\title{
Self-tuning MIMO disturbance feedforward control for active hard-mounted vibration isolators
}

\section{Citation for published version (APA):}

Beijen, M. A., Heertjes, M. F., van Dijk, J. W., \& Hakvoort, W. B. J. (2018). Self-tuning MIMO disturbance feedforward control for active hard-mounted vibration isolators. Control Engineering Practice, 72, 90-103. https://doi.org/10.1016/j.conengprac.2017.11.008

DOI:

10.1016/j.conengprac.2017.11.008

Document status and date:

Published: 01/03/2018

\section{Document Version:}

Accepted manuscript including changes made at the peer-review stage

\section{Please check the document version of this publication:}

- A submitted manuscript is the version of the article upon submission and before peer-review. There can be important differences between the submitted version and the official published version of record. People interested in the research are advised to contact the author for the final version of the publication, or visit the $\mathrm{DOI}$ to the publisher's website.

- The final author version and the galley proof are versions of the publication after peer review.

- The final published version features the final layout of the paper including the volume, issue and page numbers.

Link to publication

\section{General rights}

Copyright and moral rights for the publications made accessible in the public portal are retained by the authors and/or other copyright owners and it is a condition of accessing publications that users recognise and abide by the legal requirements associated with these rights.

- Users may download and print one copy of any publication from the public portal for the purpose of private study or research.

- You may not further distribute the material or use it for any profit-making activity or commercial gain

- You may freely distribute the URL identifying the publication in the public portal.

If the publication is distributed under the terms of Article 25fa of the Dutch Copyright Act, indicated by the "Taverne" license above, please follow below link for the End User Agreement:

www.tue.nl/taverne

Take down policy

If you believe that this document breaches copyright please contact us at:

openaccess@tue.nl

providing details and we will investigate your claim. 


\title{
Self-tuning MIMO Disturbance Feedforward Control for Active Hard-mounted Vibration Isolators
}

\author{
M.A. Beijen ${ }^{\mathrm{a}}$, M.F. Heertjes ${ }^{\mathrm{a}, \mathrm{b}}$, J. van Dijk ${ }^{\mathrm{c}}$, W.B.J. Hakvoort ${ }^{\mathrm{c}, \mathrm{d}}$ \\ ${ }^{a}$ Eindhoven University of Technology, Department of Mechanical Engineering, NL-5600 MB Eindhoven, The Netherlands \\ ${ }^{b} A S M L$, Mechatronic Systems Development, 5504 DR Veldhoven, The Netherlands \\ ${ }^{c}$ University of Twente, Department of Mechanical Automation and Mechatronics, NL-7500 AE Enschede, The Netherlands \\ ${ }^{d}$ DEMCON Advanced Mechatronics, Institutenweg 25, NL-7521 PH Enschede, The Netherlands
}

\begin{abstract}
This paper proposes a multi-input multi-output (MIMO) disturbance feedforward controller to improve the rejection of floor vibrations in active vibration isolation systems for high-precision machinery. To minimize loss of performance due to model uncertainties, the feedforward controller is implemented as a self-tuning generalized FIR filter. This filter contains a priori knowledge of the poles, such that relatively few parameters have to be estimated which makes the algorithm computationally efficient. The zeros of the filter are estimated using the filtered-error least mean squares (FeLMS) algorithm. Residual noise shaping is used to reduce bias. Conditions on convergence speed, stability, bias, and the effects of sensor noise on the self-tuning algorithm are discussed in detail. The combined control strategy is validated on a 6 -DOF Stewart platform, which serves as a multi-axis and hard-mounted vibration isolation system, and shows significant improvement in the rejection of floor vibrations.
\end{abstract}

Keywords: Active vibration isolation, MIMO feedforward control, Least mean squares optimization, High-precision mechatronics.

\section{Introduction}

Active vibration isolators are widely used to isolate highprecision machines from floor vibrations $[1,2,3]$. A classic example is the addition of skyhook damping via absolute velocity feedback to damp the suspension modes [4]. To further increase vibration isolation performance in a broad frequency range, developments are made towards high-gain feedback control $[5,6,7]$ and disturbance feedforward control $[8,9,10]$. In contrast to feedback control, where measured machine vibrations are used as controller input, feedforward control exploits measurements from external disturbances such as floor vibrations. Compared to feedback control, the advantages of feedforward control are two-fold. First, it often leads to better signal-to-noise ratios because the measured disturbance signals are not (mechanically) low-pass filtered by the passive vibration isolator itself [11]. Second, feedforward control does not affect the closed-loop stability properties, whereas highgain feedback control requires a high bandwidth that can lead to stability problems. However, disturbance feedforward control requires an accurate dynamic model of the transfer function from floor vibrations to machine vibrations, which is often difficult to obtain from modeling and identification experiments.

To suppress the problem of obtaining an accurate model in the application of disturbance feedforward control, self-

Email address: m.a.beijen@tue.nl (M.A. Beijen) tuning filters can be used to accurately estimate the controller parameters online. Often used filters in this regard are of the finite-impulse-response (FIR) type, see for example $[9,12,13,14]$. A disadvantage of FIR filters, however, is that usually many parameters are necessary to accurately describe the behavior of lightly damped resonances and low-frequency poles. This is because all FIR filter poles are located at the origin. Alternatively, one can use infinite-impulse-response (IIR) filters in which poles can be placed at locations other than the origin [15]. As a result, a more computationally efficient algorithm can be obtained in which the system can be accurately described with generally less parameters. Examples are found in [16, 17], but these works use IIR filters with self-tuning poles which can lead to instability because the poles are not bounded to the open unit disk. An alternative is found in using IIR filters with fixed poles [18, 19], also called generalized FIR filters [20]. These filters lead to a linear formulation of the estimation problem for which a unique closed-form solution exists, while the poles are not restricted to the origin.

For self-tuning filters several recursive update algorithms exist. Typical examples are least mean squares (LMS) and recursive least squares (RLS) algorithms. LMS-type algorithms are attractive because they perform relatively simple computations compared to RLS algorithms [21]. This is important for practical implementation. There are two main classes of LMS algorithms that can deal with plant dynamics in the compensation path 
(or secondary path) from actuator forces to payload accelerations. Namely, filtered-reference LMS (FxLMS) and filtered-error LMS (FeLMS), which involve filtering of the reference and the error signal with the (modeled) plant dynamics, respectively. Compared to FxLMS, FeLMS algorithms require less computational power in case of multiinput multi-output (MIMO) systems [22], but with the disadvantage of having additional requirements on the estimated plant dynamics. Hybrid methods using FeLMS [23] or Filtered-reference Filtered-error LMS (Fx-Fe-LMS) $[24,25]$ are proposed in an attempt to combine the advantages of several algorithms. However, none of the above works apply self-tuning of generalized FIR filters.

This paper has three main contributions. The first contribution is a generalization of the model-based singleinput single-output (SISO) feedforward controller from [26] to MIMO vibration isolation systems. It is shown that, despite the presence of flexible modes in the payload, the MIMO feedforward controller only depends on the damping and stiffness properties of the suspension system. Hence, an accurate estimation of these properties is necessary for the feedforward control design in achieving improved performance. The second contribution is a MIMO self-tuning algorithm that extends the SISO selftuning algorithm from [26] and combines the advantages of a low-order generalized FIR filter with the fast convergence and MIMO computational efficiency of FeLMS. This contribution also includes a detailed discussion regarding stability, convergence, and bias. Self-tuning is used to minimize uncertainty in the estimated damping and stiffness properties, hence providing the accuracy needed to maximize vibration isolation performance. The third contribution is given by the application of the self-tuning control approach to a hard-mounted vibration isolation system.

The remainder of this paper is organized as follows. The vibration isolation system, its modeling, and a feedback controller to damp the suspension modes are given in Section 2. The main problem considered in this paper is formulated in Section 3. The model-based feedforward controller is discussed in Section 4, while the self-tuning feedforward controller is described in Section 5. An experimental validation is presented in Section 6. The conclusions and main findings of this paper are given in Section 7 .

\section{Hard-mounted vibration isolation system}

The control strategy in this paper is intended for highprecision machinery with an active vibration isolation system. Figure 1a shows an experimental setup having suspension frequencies that are representative for a hardmounted isolator [27]. Compared to soft-mounted isolators, these isolators have a relatively high stiffness and suspension frequency. This setup will be used throughout this paper. The setup consists of a Stewart platform which basically comprises a mass of $5.4 \mathrm{~kg}$ connected to six voice coil motors (VCMs, type Geeplus VM4032-250) via wire springs. The VCMs provide the actuator forces

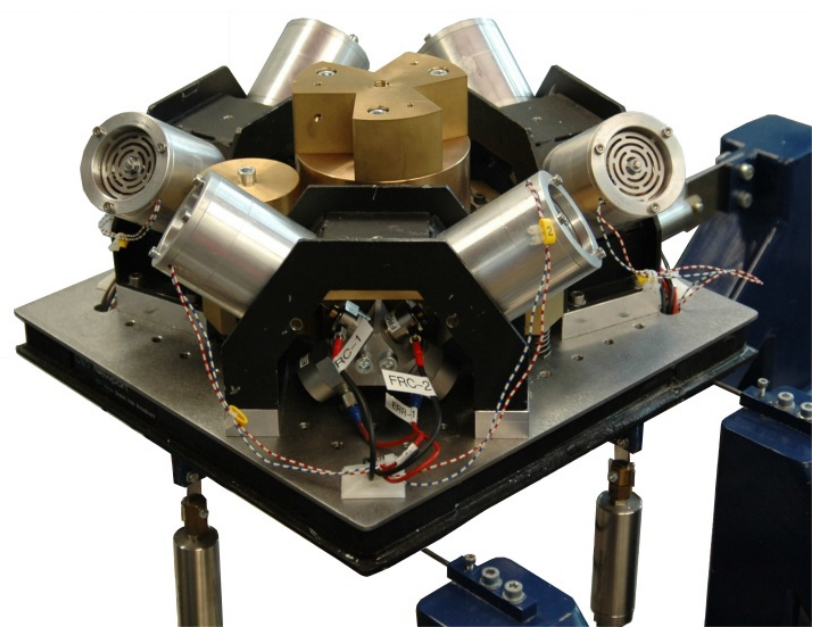

(a)

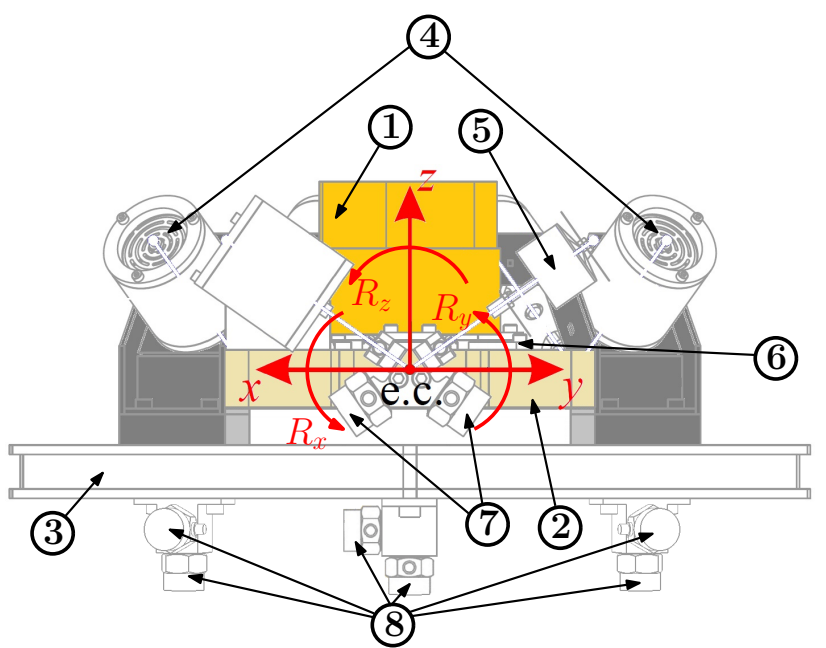

(b)

Figure 1: (a) Photo of the test setup [27]; and (b) schematic picture, including: (1) payload body, (2) platform body, (3) floor plate, (4) circular leaf springs for suspension, (5) VCMs providing actuator forces $\boldsymbol{u},(6)$ leaf springs to connect platform and payload, (7) two of the six accelerometers on the platform measuring $\ddot{\boldsymbol{q}}_{1}$, (8) accelerometers on floor plate measuring $\ddot{\boldsymbol{q}}_{\mathbf{0}}$, and (e.c.) the coordinate frame of the elastic center.

$\boldsymbol{u}(t) \in \mathbb{R}^{6}$ and are guided by circular leaf springs, the latter associate with six suspension modes of the system with resonance frequencies varying between 15 and $45 \mathrm{~Hz}$. On top of the Stewart platform a payload with a mass of $3.9 \mathrm{~kg}$ is mounted. This is done with leaf springs that give rise to additional eigenmodes in the setup at frequencies beyond $90 \mathrm{~Hz}$. The Stewart platform as a whole is mounted on a rectangular floor plate. The floor plate can be excited by three piezo stacks (type PiezoMechanik PSt 150/5/40 $\mathrm{PE}$ ) providing vibrations in $z-, R_{x}$ - and $R_{y}$-direction. The floor plate is equipped with six accelerometers (type Endevco 7703A-1000), measuring the floor plate accelerations denoted by sensor coordinates $\ddot{\boldsymbol{q}}_{\mathbf{0}}(t) \in \mathbb{R}^{6}$. Moreover, six accelerometers (type Endevco 7703A-1000) are attached to the Stewart platform, measuring the platform acceler- 
ations denoted by sensor coordinates $\ddot{\boldsymbol{q}}_{\mathbf{1}}(t) \in \mathbb{R}^{6}$. The sensors are connected to signal conditioners containing a second-order high-pass filter at $0.1 \mathrm{~Hz}$ and a second-order low-pass filter at $3000 \mathrm{~Hz}$ to filter sensor noise. The controllers are implemented on a dSPACE digital signal processor running at a sampling frequency of $f_{s}=6400 \mathrm{~Hz}$.

\subsection{Modeling}

The vibration isolation system in Figure 1 is modeled in SPACAR, which is a non-linear finite element software package for flexible multi-body dynamics [28]. For both the setup and the SPACAR model the reader is referred to [27]. The linearized equations of motion for the rigidbody model, in which the payload is assumed to be rigidly connected to the platform, are given by

$$
\begin{aligned}
\mathbf{M}_{\mathbf{r}} \ddot{x}_{\mathbf{1}}(t)+\mathbf{D}_{\mathbf{r}} \dot{x}_{\mathbf{1}}(t)+\mathbf{K}_{\mathbf{r}} \boldsymbol{x}_{\mathbf{1}}(t)= \\
\mathbf{D}_{\mathbf{0}} \dot{\boldsymbol{x}}_{\mathbf{0}}(t)+\mathbf{K}_{\mathbf{0}} \boldsymbol{x}_{\mathbf{0}}(t)+\mathbf{B} \boldsymbol{u}(t) .
\end{aligned}
$$

In this rigid-body model, $\mathbf{M}_{\mathbf{r}}, \mathbf{D}_{\mathbf{r}}, \mathbf{K}_{\mathbf{r}} \in \mathbb{R}^{6 \times 6}$ are the mass, damping-, and stiffness matrices, and $\mathbf{D}_{\mathbf{0}}, \mathbf{K}_{\mathbf{0}} \in \mathbb{R}^{6 \times 6}$ are the damping- and stiffness matrices related to the input vector $\boldsymbol{x}_{\mathbf{0}}=\left[x_{0}, y_{0}, z_{0}, R_{x 0}, R_{y 0}, R_{z 0}\right]^{T}$, which describe the floor plate displacements. In this model the hard mounts are regarded as massless elements which only induce linear damping and stiffness forces. Matrix $\mathbf{B} \in \mathbb{R}^{6 \times 6}$ describes the relation between actuator forces $\boldsymbol{u}$ and platform displacements $\boldsymbol{x}_{\mathbf{1}}=\left[x_{1}, y_{1}, z_{1}, R_{x 1}, R_{y 1}, R_{z 1}\right]^{T}$. Note that both $\boldsymbol{x}_{\mathbf{0}}$ and $\boldsymbol{x}_{\mathbf{1}}$ are expressed in the coordinate frame of the elastic center (e.c.) shown in Figure 1b, with $R_{x}, R_{y}, R_{z}$ denoting rotations about the $x, y, z$-axis of the e.c., respectively. The coordinates $\boldsymbol{x}_{\mathbf{0}}, \boldsymbol{x}_{\mathbf{1}}$ are related to the sensor coordinates $\boldsymbol{q}_{\mathbf{0}}, \boldsymbol{q}_{\mathbf{1}}$ of the experimental setup by transformation matrices $\mathbf{R}_{\mathbf{0}}, \mathbf{R}_{\mathbf{1}} \in \mathbb{R}^{6 \times 6}$, such that

$$
\boldsymbol{x}_{\mathbf{0}}(t)=\mathbf{R}_{\mathbf{0}} \boldsymbol{q}_{\mathbf{0}}(t), \quad \boldsymbol{x}_{\mathbf{1}}(t)=\mathbf{R}_{\mathbf{1}} \boldsymbol{q}_{\mathbf{1}}(t)
$$

To include the non-rigid-body part of the model, the platform mass and payload mass are split in two separate rigid bodies that are interconnected by leaf springs. By doing so, the equations of motion in (1) are augmented with six additional equations and dynamic DOFs $\boldsymbol{\Delta} \boldsymbol{q}_{\boldsymbol{f}}(t) \in \mathbb{R}^{6}$. These DOFs are related to the deformations of the leaf springs between the Stewart platform and the payload. As a result, the matrices $\mathbf{M}_{\mathbf{r}}, \mathbf{D}_{\mathbf{r}}, \mathbf{K}_{\mathbf{r}}$ at the left-hand side in (1) are augmented to become $\mathbf{M}_{\mathbf{f}}, \mathbf{D}_{\mathbf{f}}, \mathbf{K}_{\mathbf{f}} \in \mathbb{R}^{12 \times 12}$. Note that the right-hand side of (1) is augmented with zeros, since none of the inputs act directly on the payload. The augmented equations of motion are given by

$$
\begin{gathered}
\mathbf{M}_{\mathbf{f}}\left[\begin{array}{c}
\ddot{\boldsymbol{x}}_{\boldsymbol{1}}(t) \\
\boldsymbol{\Delta} \ddot{\boldsymbol{q}}_{\boldsymbol{f}}(t)
\end{array}\right]+\mathbf{D}_{\mathbf{f}}\left[\begin{array}{c}
\dot{\boldsymbol{x}}_{\boldsymbol{1}}(t) \\
\boldsymbol{\Delta} \dot{\boldsymbol{q}}_{\boldsymbol{f}}(t)
\end{array}\right]+\mathbf{K}_{\mathbf{f}}\left[\begin{array}{c}
\boldsymbol{x}_{\boldsymbol{1}}(t) \\
\boldsymbol{\Delta} \boldsymbol{q}_{\boldsymbol{f}}(t)
\end{array}\right]= \\
{\left[\begin{array}{c}
\mathbf{D}_{\mathbf{0}} \\
\mathbf{0}
\end{array}\right] \dot{\boldsymbol{x}}_{\mathbf{0}}(t)+\left[\begin{array}{c}
\mathbf{K}_{\mathbf{0}} \\
\mathbf{0}
\end{array}\right] \boldsymbol{x}_{\mathbf{0}}(t)+\left[\begin{array}{c}
\mathbf{B} \\
\mathbf{0}
\end{array}\right] \boldsymbol{u}(t) .}
\end{gathered}
$$

Using the Laplace transform, it can be derived that

$$
\begin{aligned}
{\left[\mathbf{M}_{\mathbf{f}} s^{2}+\mathbf{D}_{\mathbf{f}} s+\mathbf{K}_{\mathbf{f}}\right]\left[\begin{array}{c}
\boldsymbol{X}_{\mathbf{1}}(s) \\
\boldsymbol{\Delta} \boldsymbol{Q}_{\boldsymbol{f}}(s)
\end{array}\right]=} & \\
& {\left[\begin{array}{c}
\mathbf{D}_{\mathbf{0}} s+\mathbf{K}_{\mathbf{0}} \\
\mathbf{0}
\end{array}\right] \boldsymbol{X}_{\mathbf{0}}(s)+\left[\begin{array}{c}
\mathbf{B} \\
\mathbf{0}
\end{array}\right] \boldsymbol{U}(s), }
\end{aligned}
$$

with Laplace variable $s, \mathcal{L}\left\{\boldsymbol{x}_{\mathbf{0}}(t)\right\}=\boldsymbol{X}_{\mathbf{0}}(s), \mathcal{L}\left\{\boldsymbol{x}_{\mathbf{1}}(t)\right\}=$ $\boldsymbol{X}_{\mathbf{1}}(s), \mathcal{L}\left\{\boldsymbol{\Delta} \boldsymbol{q}_{\boldsymbol{f}}(t)\right\}=\boldsymbol{\Delta} \boldsymbol{Q}_{\boldsymbol{f}}(s)$, and $\mathcal{L}\{\boldsymbol{u}(t)\}=\boldsymbol{U}(s)$. Numerical values for the model in (4) are given in Appendix E.

\subsection{Feedback control}

Although the main focus of this paper is on feedforward control, a feedback controller is used to add skyhook damping [4] to the suspension and internal modes. Without feedback, these modes would heavily dominate the payload response and drive the system to its mechanical limits. Since the setup in Figure 1 is designed such that the $6 \times 6$ transfer function matrix from actuator forces $\boldsymbol{u}$ to sensor coordinates $\ddot{\boldsymbol{q}}_{\mathbf{1}}$ is diagonally dominant, a diagonal feedback controller is considered to be the appropriate choice. The controller is post-multiplied with $\mathbf{R}_{\mathbf{1}}^{-\mathbf{1}}$ because the transformed coordinates $\tilde{\boldsymbol{a}}_{\mathbf{1}}$ are used (instead of the sensor coordinates $\ddot{\boldsymbol{q}}_{\mathbf{1}}$ ) as feedback controller input. The feedback controller $\mathbf{C}_{\mathbf{F B}}$ is thus given by

$$
\mathbf{C}_{\mathbf{F B}}(s)=\left(\frac{\omega_{i}}{s+\omega_{i}} k_{v} \mathbf{I}_{\mathbf{6}}\right) \mathbf{R}_{\mathbf{1}}^{-1}
$$

This controller integrates the inputs (accelerations) using weak integrators with a cut-off frequency of $\omega_{i}=1 \mathrm{rad} / \mathrm{s}$ and thus provides output (feedback forces) proportional to the platform velocities needed for adding skyhook damping. Tuning of the feedback gain $k_{v}$ should be such that the suspension modes of the closed-loop system obtain sufficient relative damping, typically 0.7 . To obtain sufficient damping via feedback control that in turn guarantee sufficiently good robustness margins in practice, tuning of $k_{v}$ is based on the measured frequency response data of the system which is common practice for the considered industry. For the modeled system, $k_{v}=2000 \mathrm{Ns} / \mathrm{m}$ is used. Note that, for the aim of this paper, the actual feedback tuning is not that relevant, as long as the feedback loop provides sufficient robust stability margins, which is guaranteed by the earlier mentioned data-based feedback design, and which keeps the system within its mechanical limits. For further information regarding feedback tuning, the reader is referred to [5].

\section{Problem formulation}

In this paper, active vibration isolation of the Stewart platform will be obtained through the combination of feedforward and feedback control. The main focus in this paper is on the feedforward controller design such that the vibration isolation performance is greatly improved in a 


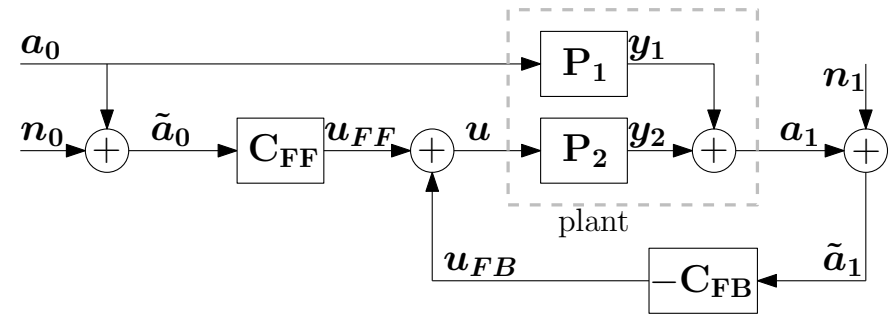

Figure 2: Combined feedforward/feedback control block diagram for active vibration isolation.

broad frequency range. As discussed in Section 2.2, feedback control is only used to damp the suspension modes and its design is not further considered in this paper.

A representative block diagram is shown in Figure 2. The input disturbance signals representing floor vibrations are denoted by $\boldsymbol{a}_{\mathbf{0}}(t) \in \mathbb{R}^{6}$ with time $t \in \mathbb{R}_{\geq 0}$. The signals representing platform vibrations are denoted by $\boldsymbol{a}_{\mathbf{1}}(t) \in \mathbb{R}^{6}$. The latter are decomposed in two parts because there are two transmission paths: signals $\boldsymbol{y}_{\mathbf{1}}(t) \in \mathbb{R}^{6}$ are caused by disturbances $\boldsymbol{a}_{\mathbf{0}}$ via the so-called primary path $\mathbf{P}_{\mathbf{1}}$, and signals $\boldsymbol{y}_{\mathbf{2}}(t) \in \mathbb{R}^{6}$ are caused by the control actions $\boldsymbol{u}(t) \in \mathbb{R}^{6}$ via the secondary path $\mathbf{P}_{\mathbf{2}}$. The control actions $\boldsymbol{u}$ are obtained by summation of the feedback controller output $\boldsymbol{u}_{\boldsymbol{F} \boldsymbol{B}}(t) \in \mathbb{R}^{6}$ and the feedforward controller output $\boldsymbol{u}_{\boldsymbol{F} \boldsymbol{F}}(t) \in \mathbb{R}^{6}$ induced by the controllers $\mathbf{C}_{\mathbf{F B}}$ and $\mathbf{C}_{\mathbf{F F}}$, which react on the measured platform vibrations and the floor vibrations respectively. The signals $\tilde{\boldsymbol{a}}_{\mathbf{0}}(t), \tilde{\boldsymbol{a}}_{\mathbf{1}}(t) \in \mathbb{R}^{6}$ represent measurements of $\boldsymbol{a}_{\mathbf{0}}, \boldsymbol{a}_{\mathbf{1}}$ with additive sensor noise signals $\boldsymbol{n}_{\mathbf{0}}(t), \boldsymbol{n}_{\mathbf{1}}(t) \in \mathbb{R}^{6}$, respectively.

Transfer functions for $\mathbf{P}_{\mathbf{1}}$ and $\mathbf{P}_{\mathbf{2}}$ are obtained from the model in Section 2. Since the setup is equipped with accelerometers, one can define $s^{2} \boldsymbol{X}_{\mathbf{0}}(s)=\boldsymbol{A}_{\mathbf{0}}(s)$, $s^{2} \boldsymbol{X}_{\mathbf{1}}(s)=\boldsymbol{A}_{\mathbf{1}}(s)$. Then, using (4) and Figure 2 the inputoutput equations are obtained as

$$
\boldsymbol{A}_{\mathbf{1}}(s)=\mathbf{P}_{\mathbf{1}}(s) \boldsymbol{A}_{\mathbf{0}}(s)+\mathbf{P}_{\mathbf{2}}(s) \boldsymbol{U}(s),
$$

with

$$
\begin{aligned}
& \mathbf{P}_{\mathbf{1}}(s)=\mathbf{G}(s)\left[\mathbf{D}_{\mathbf{0}} s+\mathbf{K}_{\mathbf{0}}\right] \\
& \mathbf{P}_{\mathbf{2}}(s)=s^{2} \mathbf{G}(s) \mathbf{B}
\end{aligned}
$$

where $\mathbf{G}(s)$ represents the common terms in $\mathbf{P}_{\mathbf{1}}$ and $\mathbf{P}_{\mathbf{2}}$ which are given by

$$
\mathbf{G}(s)=\left[\begin{array}{ll}
\mathbf{I}_{\mathbf{6}} & \mathbf{0}
\end{array}\right]\left[\mathbf{M}_{\mathbf{f}} s^{2}+\mathbf{D}_{\mathbf{f}} s+\mathbf{K}_{\mathbf{f}}\right]^{-1}\left[\begin{array}{c}
\mathbf{I}_{\mathbf{6}} \\
\mathbf{0}
\end{array}\right]
$$

Since $\boldsymbol{U}(s)=\mathbf{C}_{\mathbf{F F}}(s) \tilde{\boldsymbol{A}}_{\mathbf{0}}(s)-\mathbf{C}_{\mathbf{F B}}(s) \tilde{\boldsymbol{A}}_{\mathbf{1}}(s)$ it follows that (6) can be rewritten:

$$
\boldsymbol{A}_{\mathbf{1}}(s)=\mathcal{T}(s) \boldsymbol{A}_{\mathbf{0}}(s)+\mathcal{S}_{0}(s) \boldsymbol{N}_{\mathbf{0}}(s)+\mathcal{S}_{1}(s) \boldsymbol{N}_{\mathbf{1}}(s)
$$

with $\mathcal{L}\left\{\boldsymbol{a}_{\mathbf{1}}(t)\right\}=\boldsymbol{A}_{\mathbf{1}}(s), \mathcal{L}\left\{\boldsymbol{a}_{\mathbf{0}}(t)\right\}=\boldsymbol{A}_{\mathbf{0}}(s), \mathcal{L}\left\{\boldsymbol{n}_{\mathbf{0}}(t)\right\}=$
$\boldsymbol{N}_{\mathbf{0}}(s), \mathcal{L}\left\{\boldsymbol{a}_{\mathbf{1}}(t)\right\}=\boldsymbol{A}_{\mathbf{1}}(s)$, and

$$
\begin{aligned}
\mathcal{T}(s) & =\mathbf{S}_{\mathbf{F B}}(s)\left(\mathbf{P}_{\mathbf{1}}(s)+\mathbf{P}_{\mathbf{2}}(s) \mathbf{C}_{\mathbf{F F}}(s)\right), \\
\mathcal{S}_{0}(s) & =\mathbf{S}_{\mathbf{F B}}(s) \mathbf{P}_{\mathbf{2}}(s) \mathbf{C}_{\mathbf{F F}}(s), \\
\mathcal{S}_{1}(s) & =-\mathbf{S}_{\mathbf{F B}}(s) \mathbf{P}_{\mathbf{2}}(s) \mathbf{C}_{\mathbf{F B}}(s),
\end{aligned}
$$

where the feedback sensitivity function $\mathbf{S}_{\mathbf{F B}}$ is defined as

$$
\mathbf{S}_{\mathbf{F B}}(s)=\left(\mathbf{I}_{\mathbf{6}}+\mathbf{P}_{\mathbf{2}}(s) \mathbf{C}_{\mathbf{F B}}(s)\right)^{-1} .
$$

In $(10), \mathcal{T}(s)$ represents the transmissibility function matrix that describes the input-output relation between $\boldsymbol{a}_{\mathbf{0}}$ and $\boldsymbol{a}_{1}$, while $\mathcal{S}_{0}(s), \mathcal{S}_{1}(s)$ represent the noise sensitivity functions with respect to $\boldsymbol{n}_{\mathbf{0}}$ and $\boldsymbol{n}_{\mathbf{1}}$, respectively.

In general, the control objective in active vibration isolation is to minimize the platform vibrations $\boldsymbol{a}_{\mathbf{1}}$, which implies a trade-off between minimizing $\mathcal{T} \boldsymbol{A}_{\mathbf{0}}, \mathcal{S}_{0} \boldsymbol{N}_{\mathbf{0}}$, and $\mathcal{S}_{1} \boldsymbol{N}_{\mathbf{1}}$ in (9). In the absence of sensor noise, i.e. $\boldsymbol{N}_{\mathbf{0}}=$ $\boldsymbol{N}_{\mathbf{1}}=\mathbf{0}$, minimization of $\boldsymbol{A}_{\mathbf{1}}$ in (9) reduces to minimization of $\mathcal{T}$. In that case, it is derived from (10) that the ideal (model-based) feedforward controller is given by

$$
\mathbf{C}_{\mathbf{F F}}(s)=-\mathbf{P}_{\mathbf{2}}^{-\mathbf{1}}(s) \mathbf{P}_{\mathbf{1}}(s) .
$$

Hence, substitution of (12) in (10) results in $\mathcal{T}=\mathbf{0}$ such that $\boldsymbol{A}_{\mathbf{1}}=\mathbf{0}$ in (9) in the case that $\boldsymbol{N}_{\mathbf{0}}=\boldsymbol{N}_{\mathbf{1}}=\mathbf{0}$. The controller in (12) is known as the Wiener controller [16], and is used in Section 4 to derive a model-based controller. To minimize performance loss due to model uncertainty, and to account for sensor noise, the model-based controller will be turned into a self-tuning controller in Section 5 . Note that without control, i.e. $\mathbf{C}_{\mathbf{F B}}=\mathbf{C}_{\mathbf{F F}}=\mathbf{0}, \mathcal{T}$ in (10) equals $\mathbf{P}_{\mathbf{1}}$.

\section{Model-based feedforward control}

In this section, a model-based feedforward controller is derived for the model in Section 2.1 such that the transmissibility is lowered for frequencies beyond $2 \mathrm{~Hz}$. First, the model-based controller design is discussed. Second, practical aspects are discussed that limit performance and impede a straightforward implementation of the modelbased feedforward controller.

\subsection{Model-based controller design}

Recall from (12) that perfect cancellation of floor vibrations is obtained when the feedforward controller is designed as $\mathbf{C}_{\mathbf{F F}}(s)=\mathbf{P}_{\mathbf{2}}^{-\mathbf{1}}(s) \mathbf{P}_{\mathbf{1}}(s)$. When the expressions for $\mathbf{P}_{\mathbf{1}}$ and $\mathbf{P}_{\mathbf{2}}$ from (7) are substituted in (12) it is obtained that

$$
\mathbf{C}_{\mathbf{F F}, \mathbf{o p t}}(s)=-\frac{1}{s^{2}}(\overline{\mathbf{D}} s+\overline{\mathbf{K}})
$$

with

$$
\overline{\mathbf{D}}=\mathbf{B}^{-1} \mathbf{D}_{\mathbf{0}}, \quad \overline{\mathbf{K}}=\mathbf{B}^{-1} \mathbf{K}_{\mathbf{0}} .
$$




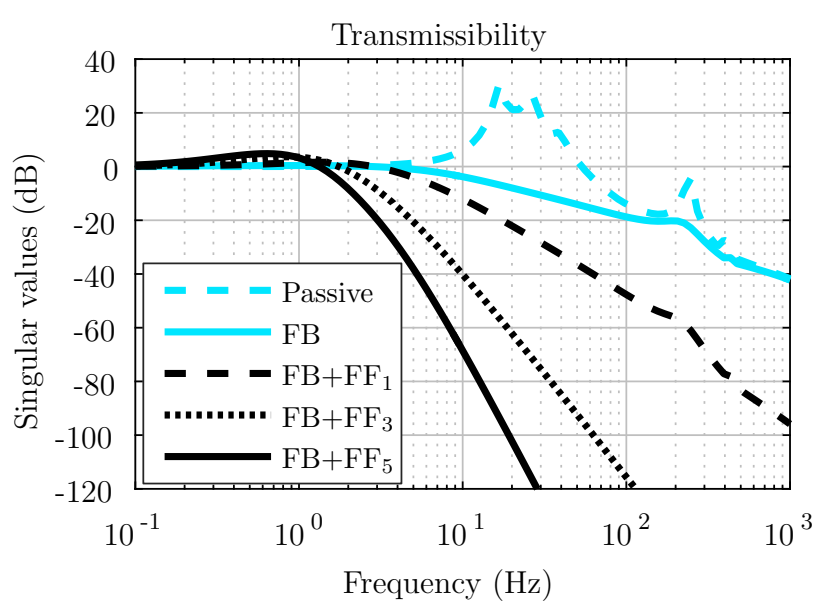

Figure 3: Maximum singular values of $\mathcal{T}$ for the six-axis vibration isolation system, in case of the passive system (dashed blue), the feedback (FB) controlled system using (5) (solid blue), and the combined feedback plus model-based feedforward (FF) controlled system using (5) and (15) with $\alpha=2 \times 2 \pi \mathrm{rad} / \mathrm{s}$ and $n=1,3,5$ (black).

Note that in (14) it is assumed that $\mathbf{B}$ is invertible, which is reasonable since the actuators of the system in Figure 1 are placed such that the Stewart platform can be actuated in all directions. Moreover, observe from (13) that $\mathbf{C}_{\mathbf{F F} \text {,opt }}$ only requires information from the rigid-body model to obtain perfect cancellation of floor vibrations despite the presence of internal non-rigid-body modes. In other words, the ideal feedforward controller is independent of the internal modes of the payload. From a physical point of view, the ideal feedforward controller perfectly compensates the disturbance forces transmitted through the hard mounts, such that the net forces on the platform become zero.

In practice, the controller in (13) is not feasible because it induces drift and actuator saturation as a result of using pure integrators. To cope with this problem, the pure integrators are replaced by $n^{\text {th }}$-order weak integrators. That is, the integrating actions are cut off below a certain frequency $\alpha$. By doing so, an approximate Wiener controller is given by

$$
\mathbf{C}_{\mathbf{F F}, n}(s)=-H_{(\alpha, n)}^{2}(s)(\overline{\mathbf{D}} s+\overline{\mathbf{K}})
$$

with

$$
H_{(\alpha, n)}(s)=\frac{1-L_{(\alpha, n)}(s)}{s},
$$

that describes an $n^{\text {th }}$-order weak integrator with relative degree one, and

$$
L_{(\alpha, n)}(s)=\left(\frac{\alpha}{s+\alpha}\right)^{n},
$$

representing an $n^{\text {th }}$-order low-pass filter with cut-off frequency $\alpha$. The feedforward controller in (15) can be

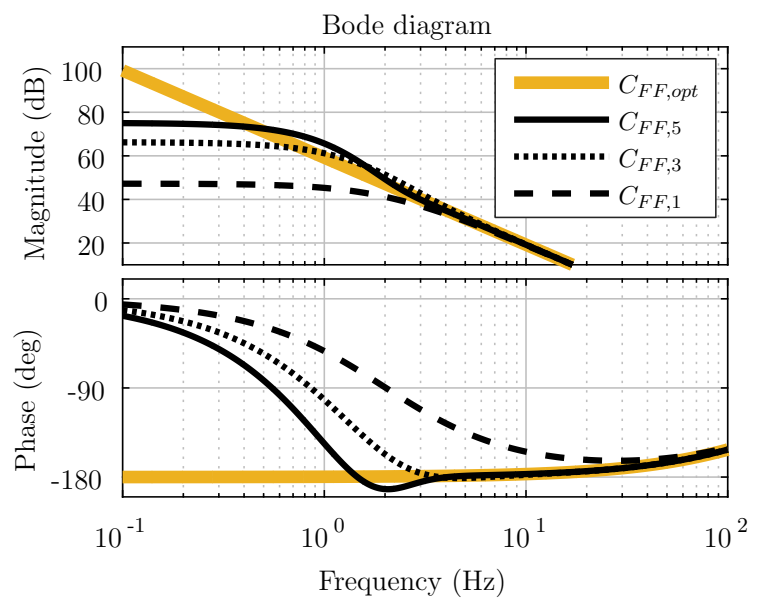

Figure 4: Bode plots of the $(1,1)$-components, i.e. the controller transfer function from input 1 to output 1 , in $\mathbf{C}_{\mathbf{F F}, 1}, \mathbf{C}_{\mathbf{F F}, 3}$, and $\mathbf{C}_{\mathbf{F F}, 5}$ compared to the $(1,1)$-component in $\mathbf{C}_{\mathbf{F F} \text {,opt }}$. Increasing $n$ results in a trade-off: (a) a better approximation of the phase with respect to $\mathbf{C}_{\mathbf{F F} \text {,opt }}$, but (b) increased controller gain for low frequencies.

rewritten as

$$
\begin{aligned}
\mathbf{C}_{\mathbf{F F}, n}(s) & =-\left(\frac{1-L_{(\alpha, n)}(s)}{s}\right)^{2}(\overline{\mathbf{D}} s+\overline{\mathbf{K}}) \\
& =-\left(1-L_{(\alpha, n)}(s)\right)^{2} \frac{1}{s^{2}}(\overline{\mathbf{D}} s+\overline{\mathbf{K}}) \\
& =-\left(1-L_{(\alpha, n)}(s)\right)^{2} \mathbf{P}_{\mathbf{2}}^{-\mathbf{1}}(s) \mathbf{P}_{\mathbf{1}}(s) .
\end{aligned}
$$

Substitution of (18) in (10) and after some algebra gives

$$
\mathcal{T}(s)=\left(2-L_{(\alpha, n)}(s)\right) L_{(\alpha, n)}(s) \mathbf{S}_{\mathbf{F B}}(s) \mathbf{P}_{\mathbf{1}}(s),
$$

with $\mathbf{S}_{\mathbf{F B}}(s) \mathbf{P}_{\mathbf{1}}(s)$ representing the primary path in case the feedback loop is closed, see (7) and (11). For $s=j \omega$ and $\omega>\alpha,(19)$ reduces to

$$
\mathcal{T}(j \omega) \rightarrow 2 L_{(\alpha, n)}(j \omega) \mathbf{S}_{\mathbf{F B}}(j \omega) \mathbf{P}_{\mathbf{1}}(j \omega),
$$

because $L_{(\alpha, n)}$ dominates over $L_{(\alpha, n)}^{2}$ for $\omega>\alpha$. Eq. (20) shows that, potentially, a transmissibility function with arbitrary roll-off can be created for frequencies $\omega>\alpha$ by increasing the amount of roll-off in $L_{(\alpha, n)}$. By means of example, Figure 3 shows various plots of $\mathcal{T}$ through singular values. Three cases of feedforward control with $n=1,3,5$ and $\alpha=2 \times 2 \pi \mathrm{rad} / \mathrm{s}(2 \mathrm{~Hz})$ are depicted. Beyond the frequency $\alpha$, the figure clearly shows a reduction of $\mathcal{T}$ with a decay of $n \times 20 \mathrm{~dB} /$ decade. Note that for frequencies smaller than $\alpha$, performance is slightly deteriorated with respect to the passive system. This performance deterioration could be reduced by replacing $H_{(\alpha, n)}^{2}$ in (15) by $H_{\left(\alpha_{1}, n\right)} H_{\left(\alpha_{2}, n\right)}$ with $\alpha_{1}>\alpha_{2}$, i.e. using different values of $\alpha$. However, this solution direction is not pursued in order to keep clarity of presentation.

To illustrate the behavior of the controllers containing $n^{\text {th }}$-order weak integrators in terms of Bode plots, Figure 4 shows a comparison between the $(1,1)$-components 
of $\mathbf{C}_{\mathbf{F F}, 1}, \mathbf{C}_{\mathbf{F F}, 3}$, and $\mathbf{C}_{\mathbf{F F}, 5}$ and the corresponding com-

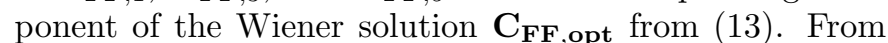
Figure 4 it can be observed that increasing $n$ leads to less phase shifts in $\mathbf{C}_{\mathbf{F F}, n}$ with respect to $\mathbf{C}_{\mathbf{F F} \text {,opt }}$ beyond the cut-off frequency $\alpha(2 \mathrm{~Hz})$ and, therefore, improved roll-off in $\mathcal{T}$ for $\omega>\alpha$ such as shown in Figure 3. However, increasing $n$ comes at the expense of a larger controller gain for the lower frequency interval,

$$
\mathbf{C}_{\mathbf{F F}, n}(0)=-\left(\frac{n}{\alpha}\right)^{2} \overline{\mathbf{K}}
$$

which is undesired because of drift and actuator saturation. Note that (21) can be derived from the static gain of the weak integrator, or

$$
\lim _{s \rightarrow 0} H_{(\alpha, n)}(s)=\lim _{s \rightarrow 0} \frac{1-L_{(\alpha, n)}(s)}{s}=\frac{n}{\alpha} .
$$

\subsection{Practical aspects}

The theoretical performance improvements shown in Figure 3 will not easily be obtained in practice. Important performance limiters in practice are actuator-floor interaction, sensor filtering, and parameter uncertainty, which are discussed below.

\subsubsection{Actuator-floor interaction}

In this paper, it is assumed that the reaction forces of the actuator exerted on the floor plate do not influence $\ddot{\boldsymbol{q}}_{\mathbf{0}}$. For the given setup, this is a valid assumption since both the control forces and guidance stiffnesses of the VCMs are small compared to the piezo shaker forces and piezo and floor plate stiffnesses. In general, however, the floor acceleration might be significantly influenced by the reaction forces of the actuators. This can result in a possibly unstable feedback loop from the reaction forces to the floor plate accelerometers. An extensive discussion about this topic is found in, e.g., [17]. However, for the setup considered in this paper, no stability problems occurred during the measurements. Therefore, this stability aspect is not further addressed.

\subsubsection{Sensor filtering}

As discussed in Section 2, the signal conditioners for the sensor signals contain a $0.1 \mathrm{~Hz}$ high-pass filter and a $3000 \mathrm{~Hz}$ low-pass filter to suppress sensor noise, internal sensor dynamics, and the effects of aliasing. To analyze performance including the effects of sensor filtering, $\mathbf{P}_{\mathbf{2}}$ in (7) is extended to

$$
\mathbf{P}_{\mathbf{2}, \text { new }}=\left(\frac{s^{2}}{s^{2}+2 \zeta_{1} \omega_{1} s+\omega_{1}^{2}}\right)\left(\frac{\omega_{2}^{2}}{s^{2}+2 \zeta_{2} \omega_{2} s+\omega_{2}^{2}}\right) \mathbf{P}_{\mathbf{2}}
$$

with $\omega_{1}=0.1 \times 2 \pi \mathrm{rad} / \mathrm{s}, \omega_{2}=3000 \times 2 \pi \mathrm{rad} / \mathrm{s}$ representing the filter frequencies, and $\zeta_{1}=\zeta_{2}=0.7$ representing the relative damping coefficients of the filters. The corresponding transmissibility is plotted in Figure 5. The figure clearly shows that sensor filtering deteriorates performance

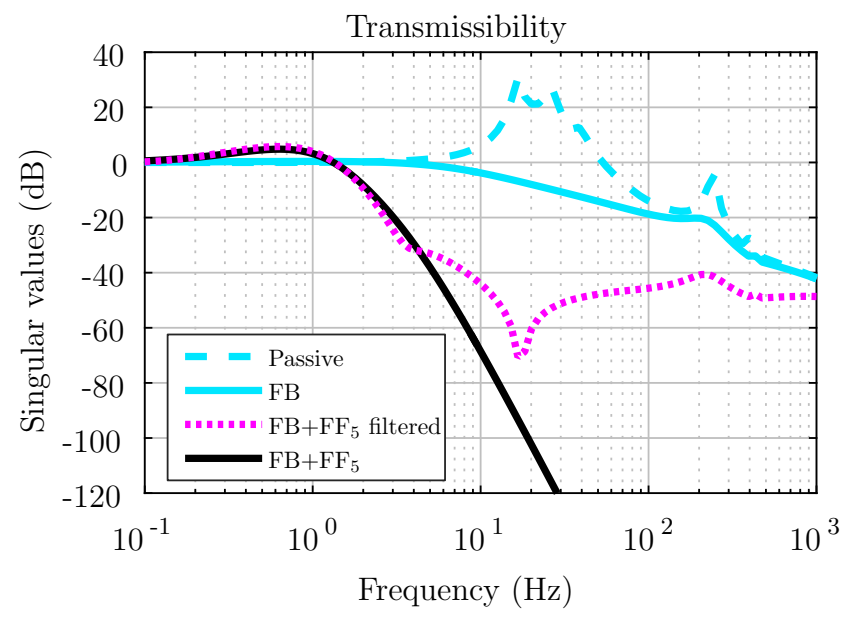

Figure 5: Maximum singular values of $\mathcal{T}$ for the six-axis vibration isolation system. The blue and black lines are identical to the lines in Figure 3. The magenta dotted line shows that disturbance suppression in terms of $\mathcal{T}$ is limited when including sensor filtering, see Section 4.2.2.

for frequencies beyond $4 \mathrm{~Hz}$, even though the high cut-off frequency $\omega_{2}$ in the sensor filter is more than 700 times higher. Below $4 \mathrm{~Hz}$, the effect of sensor filtering on performance deterioration is hardly visible. This is because the lower cut-off frequency $\omega_{1}$ in the sensor filter is much lower than the cut-off frequency $\alpha(2 \mathrm{~Hz})$ in the weak integrators which already limit performance improvements at low frequencies.

\subsubsection{Parameter uncertainty}

Performance as discussed in Figure 3 is obtained in case the feedforward controller parameters exactly match the plant parameters. However, in practice it is often difficult to accurately identify $\mathbf{P}_{\mathbf{1}}$, hence $\overline{\mathbf{D}}, \overline{\mathbf{K}}$ in (15), because it is often not possible to excite the floor sufficiently. As a result, the controller parameters will not match the plant parameters exactly. For example, Figure 6 shows the maximum singular values of the transmissibility function in case of a $1 \%$ and $10 \%$ parameter estimation error, i.e. $\overline{\mathbf{D}} \rightarrow 0.99 \overline{\mathbf{D}}, \overline{\mathbf{K}} \rightarrow 0.99 \overline{\mathbf{K}}$ and $\overline{\mathbf{D}} \rightarrow 0.9 \overline{\mathbf{D}}, \overline{\mathbf{K}} \rightarrow 0.9 \overline{\mathbf{K}}$, respectively. It follows that the maximum achievable performance is proportional with the parameter estimation error. For example, when there is an estimation error of $1 \%$, the reduction in transmissibility is also limited to $1 \%$ (or $0.01=-40 \mathrm{~dB}$ ) with respect to performance without feedforward control. It thus makes sense to minimize parameter uncertainty, which is the aim in the next section by applying self-tuning feedforward control.

\section{Self-tuning feedforward control}

The motivation for residing to self-tuning methods is to minimize performance limitations due to parameter uncertainty, see Section 4.2.3. The first step in self-tuning controller design is to rewrite the model-based feedforward 


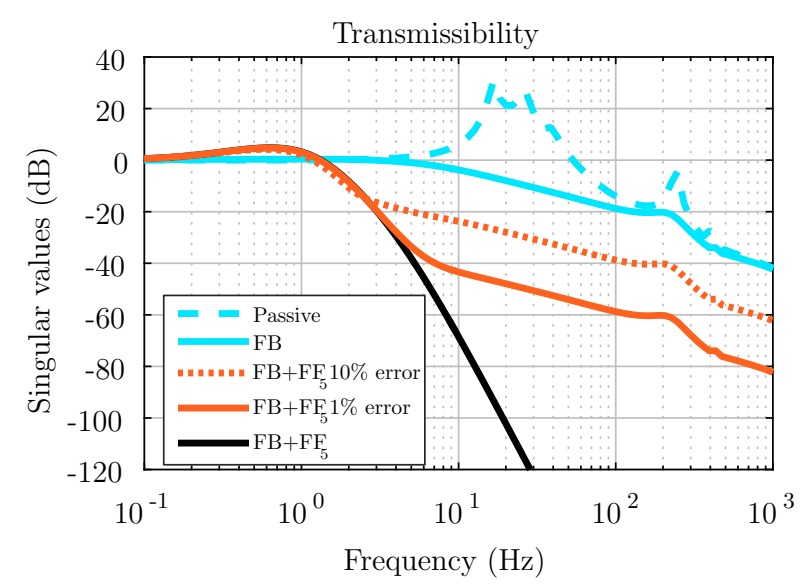

Figure 6: Maximum singular values of $\mathcal{T}$ for the six-axis vibration isolation system. The blue and black lines are identical to the lines in Figure 3. The red lines show that disturbance suppression in terms of $\mathcal{T}$ is limited when having parameter estimation errors, see Section 4.2.3.

controller from Section 4 to a generalized FIR filter structure, which is presented in Section 5.1. The second step is to determine the update law for the controller parameters, which is derived in Section 5.2. A convergence analysis and simulation studies for the self-tuning controller are presented in Section 5.3 and Section 5.4, respectively.

\subsection{Generalized FIR filter structure}

In this paper, the generalized FIR filter structure is used for self-tuning feedforward control. In this structure, an explicit separation between the zeros and the poles of the controller is made. The poles are fixed a priori in so-called basis functions. The zeros are subsequently determined online by a self-tuning algorithm. This section shows how the generalized FIR filter structure can be derived from the model-based controller in (15). Defining $\tilde{\boldsymbol{A}}_{\mathbf{0}}(s) \in \mathbb{C}^{6}$ as the Laplace transform of $\tilde{\boldsymbol{a}}_{\mathbf{0}}$ in Figure 2, the output of a feedforward controller similar to (15) can be written as

$$
\boldsymbol{U}_{\boldsymbol{F F}}(s)=\left[\begin{array}{ll}
-\overline{\mathbf{D}} & -\overline{\mathbf{K}}
\end{array}\right]\left[\begin{array}{c}
H_{(\alpha, n)}(s) \tilde{\boldsymbol{A}}_{\mathbf{0}}(s) \\
H_{(\alpha, n)}^{2}(s) \tilde{\boldsymbol{A}}_{\mathbf{0}}(s)
\end{array}\right]
$$

Since the self-tuning algorithm operates in discrete-time at sampling frequency $f_{s}$, a discrete-time representation of (23) is used which reads

$$
\boldsymbol{u}_{\boldsymbol{F F}}(k)=\left[\begin{array}{ll}
-\overline{\mathbf{D}}_{\mathbf{d}} & -\overline{\mathbf{K}}_{\mathbf{d}}
\end{array}\right]\left[\begin{array}{c}
H_{(\alpha, n)}(q) \tilde{\boldsymbol{a}}_{\mathbf{0}}(k) \\
H_{(\alpha, n)}^{2}(q) \tilde{\boldsymbol{a}}_{\mathbf{0}}(k)
\end{array}\right],
$$

with $\overline{\mathbf{D}}_{\mathbf{d}}, \overline{\mathbf{K}}_{\mathbf{d}} \in \mathbb{R}^{6 \times 6}$ containing the discrete-time controller parameters, $q$ representing the backward shift operator, and $k \in \mathbb{N}$ referring to time samples $t_{k}=k T_{s}$ with sampling time $T_{s}=1 / f_{s}$. To improve the convergence properties of the update algorithm, (24) is rewritten as

$$
\boldsymbol{u}_{\boldsymbol{F} \boldsymbol{F}}(k)=H_{(\alpha, n)}(q) \underbrace{\left[\begin{array}{ll}
-\overline{\mathbf{D}}_{\mathbf{d}} & \frac{-1}{\beta} \overline{\mathbf{K}}_{\mathbf{d}}
\end{array}\right]}_{\mathbf{W}^{*}} \underbrace{\left[\begin{array}{c}
\tilde{\boldsymbol{a}}_{\mathbf{0}}(k) \\
\beta H_{(\alpha, n)}(q) \tilde{\boldsymbol{a}}_{\mathbf{0}}(k)
\end{array}\right]}_{\tilde{\boldsymbol{\psi}}(k)} .
$$

In (25), $\beta \in \mathbb{R}$ is a scaling constant (Section 5.3.2), the matrix $\mathbf{W}^{*} \in \mathbb{R}^{6 \times 12}$ contains the model-based controller parameters, and $\tilde{\psi}(k) \in \mathbb{R}^{12}$ represents the so-called regression vector. To find (online) the parameters in $\mathbf{W}^{*}$, which are assumed to be a priori unknown, (25) is rewritten such that the parameters from $\mathbf{W}^{*}$ are stored in a single column vector $\boldsymbol{w}^{*}$, or

$$
\begin{aligned}
& \boldsymbol{u}_{\boldsymbol{F F}}(k)=H_{(\alpha, n)}(q) \cdot \\
& \underbrace{\left[\begin{array}{ccc}
\tilde{\boldsymbol{\psi}}^{T}(k) & \ldots & \mathbf{0} \\
\vdots & \ddots & \vdots \\
\mathbf{0} & \ldots & \tilde{\boldsymbol{\psi}}^{T}(k)
\end{array}\right]}_{\tilde{\tilde{\Psi}}(k)} \underbrace{\left[\begin{array}{c}
\left(\mathbf{W}_{(1,:)}^{*}\right)^{T} \\
\vdots \\
\left(\mathbf{W}_{(6,:)}^{*}\right)^{T}
\end{array}\right]}_{\boldsymbol{w}^{*}},
\end{aligned}
$$

with regression matrix $\tilde{\boldsymbol{\Psi}}(k) \in \mathbb{R}^{6 \times 72}$, parameter vector $\boldsymbol{w}^{*} \in \mathbb{R}^{72}$ containing the model-based controller parameters, and $\left(\mathbf{W}_{(i,:)}^{*}\right)^{T}$ denoting the transposed $i^{\text {th }}$ row of $\mathbf{W}^{*}$. Eq. (26) turns into a self-tuning controller if the modelbased parameter vector $\boldsymbol{w}^{*}$ is replaced by the time-varying vector $\boldsymbol{w}(k)$ that is estimated online:

$$
\boldsymbol{u}_{\boldsymbol{F F}}(k)=H_{(\alpha, n)}(q) \tilde{\boldsymbol{\Psi}}(k) \boldsymbol{w}(k) .
$$

Since (27) is affine in the parameters, minimization of a quadratic cost criterion to estimate $\boldsymbol{w}(k)$ renders a convex optimization problem that (under certain conditions) has a unique closed-form solution. The poles of the self-tuning filter are fixed in $\tilde{\boldsymbol{\psi}}$ such that adaptation of $\boldsymbol{w}(k)$ only involves estimation of its zeros, which makes that this filter has the structure of a generalized FIR filter [20].

\subsection{Update law using the FeLMS algorithm}

The update law for $\boldsymbol{w}(k)$ will be based on the filterederror least mean squares (FeLMS) algorithm [23] combined with residual noise shaping $[29,30]$. As mentioned earlier, FeLMS is attractive because it combines relatively low computational power with good convergence properties. Residual noise shaping is used to add frequency weighting to the error signal that is minimized.

The optimization algorithm [31] uses the steepest descent method [21] with corresponding update law

$$
\boldsymbol{w}(k+1)=\boldsymbol{w}(k)-\frac{\mu(k)}{2}\left(\frac{\partial J(k)}{\partial \boldsymbol{w}}\right)^{T}
$$

with adaptation rate $\mu(k)$, which will be determined in Section 5.3.1, and gradient $\frac{\partial J(k)}{\partial \boldsymbol{w}}$ of a scalar-valued cost 
function $J$ to be minimized. The parameters are updated at every time sample $t_{k}$. The cost function $J$ is chosen as the squared filtered-error at time sample $t_{k}$, or

$$
J(k)=\boldsymbol{e}^{T}(k) \boldsymbol{e}(k),
$$

such that

$$
\left(\frac{\partial J(k)}{\partial \boldsymbol{w}}\right)^{T}=\left(\frac{\partial J(k)}{\partial \boldsymbol{e}} \frac{\partial \boldsymbol{e}(k)}{\partial \boldsymbol{w}}\right)^{T}=2\left(\frac{\partial \boldsymbol{e}(k)}{\partial \boldsymbol{w}}\right)^{T} \boldsymbol{e}(k),
$$

with filtered error $\boldsymbol{e}(k) \in \mathbb{R}^{6}$ obtained from the measured output signal $\tilde{\boldsymbol{a}}_{\mathbf{1}}$ (see Figure 2 ) by

$$
\begin{aligned}
\boldsymbol{e}(k) & =H_{(\alpha, n)}^{-1}(q) \mathbf{N}(q) \hat{\mathbf{P}}_{\mathbf{2}}^{-1}(q) \tilde{\boldsymbol{a}}_{\mathbf{1}}(k) \\
& =H_{(\alpha, n)}^{-1}(q) \mathbf{N}(q) \hat{\mathbf{P}}_{\mathbf{2}}^{-1}(q)\left(\boldsymbol{y}_{\mathbf{1}}(k)+\boldsymbol{y}_{\mathbf{2}}(k)+\boldsymbol{n}_{\mathbf{1}}(k)\right)
\end{aligned}
$$

This expression for $\boldsymbol{e}(k)$ can be explained as follows. From FeLMS [13], it is known that $\tilde{\boldsymbol{a}}_{\mathbf{1}}$ must be filtered by the estimated inverse ${ }^{1}$ of the secondary path, denoted by $\hat{\mathbf{P}}_{\mathbf{2}}^{-1}$, and by $H_{(\alpha, n)}^{-1}$ to compensate for the weak integrator used as pre-multiplication filter in (25). Residual noise shaping [29] introduces an additional filter $\mathbf{N}$ to add frequency weighting. Design considerations for $\mathbf{N}$ will be further discussed in Section 5.3.3, while the actual design of $\mathbf{N}$ is considered in Section 5.4. Under the assumption of slow adaptation of the weights, and $\hat{\mathbf{P}}_{\mathbf{2}}(q)=\mathbf{P}_{\mathbf{2}}(q)$, Appendix A shows that the gradient in (30) can be estimated by

$$
\left(\frac{\partial J(k)}{\partial \boldsymbol{w}}\right)^{T} \approx 2[\mathbf{N}(q) \tilde{\boldsymbol{\Psi}}(k)]^{T} \boldsymbol{e}(k) .
$$

Note that the assumption $\hat{\mathbf{P}}_{\mathbf{2}}=\mathbf{P}_{\mathbf{2}}$ has consequences for convergence and stability which is further addressed in Section 5.3. Substitution of (33) in (28) gives the (approximated) update law

$$
\boldsymbol{w}(k+1)=\boldsymbol{w}(k)-\mu(k)[\mathbf{N}(q) \tilde{\boldsymbol{\Psi}}(k)]^{T} \boldsymbol{e}(k)
$$

An overview of the generalized FIR filter with residual noise shaping is shown in Figure $7 . \mathbf{C}_{\mathbf{F F}}$ includes the control action from $(27)$ with $\boldsymbol{w}(k)$ determined using the update law in (34).

Remark 1. The self-tuning controller implementation in Figure 7 needs the inverse of the estimated secondary path, or $\hat{\mathbf{P}}_{\mathbf{2}}^{-1}$, which generally becomes non-proper. To enable a causal implementation of the proposed FeLMS algorithm in that case, a solution is found in adding poles to the filter $\mathbf{N}$ that effectively make the product $H_{(\alpha, n)}^{-1} \mathbf{N}_{\hat{\mathbf{P}}_{\mathbf{2}}^{-1}}$ in Figure 7 proper. Also, $\hat{\mathbf{P}}_{\mathbf{2}}$ may contain transmission zeros outside

\footnotetext{
${ }^{1}$ Remarks on a causal and stable implementation of $\hat{\mathbf{P}}_{\mathbf{2}}^{-1}$ are given in Remark 1.
}

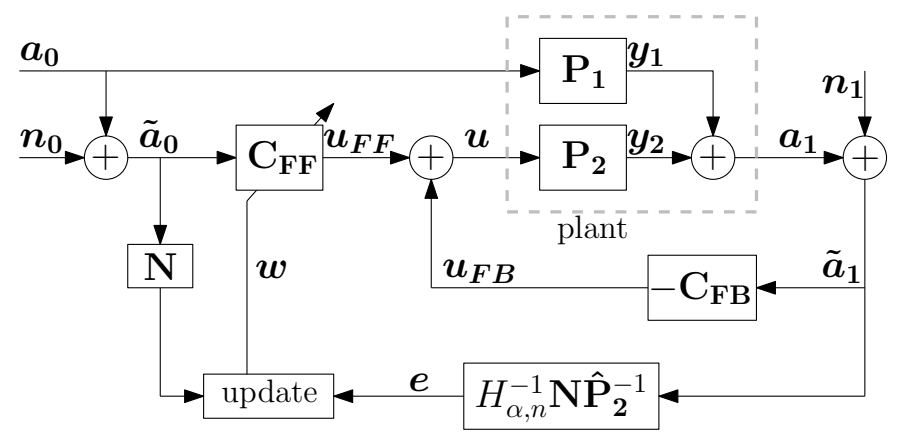

Figure 7: Implementation of the generalized FIR filter.

the unit disk, that render $\hat{\mathbf{P}}_{\mathbf{2}}^{-1}$ unstable. There are two possible solutions for this problem. First, the sensors and actuators can be relocated. Second, modified versions of the FeLMS can be used. An example is the Filtered-reference Filtered-error LMS (Fx-Fe-LMS) method [25, 24], in which the error signals are only filtered by the invertible (and minimum-phase) part of $\hat{\mathbf{P}}_{\mathbf{2}}^{-1}$, while the reference signals $\tilde{\boldsymbol{a}}_{\mathbf{0}}$ are filtered by the non-minimum phase part of $\hat{\mathbf{P}}_{\mathbf{2}}$. Another alternative is using hybrid Filtered-error LMS [23], which combines inversion-based FeLMS [32, 31] with adjoint FeLMS. Using this combination, the filter can be constructed by taking the inverse of the minimum-phase part of $\hat{\mathbf{P}}_{2}$ and the adjoint of the maximum-phase part of $\hat{\mathbf{P}}_{\mathbf{2}}$.

\subsection{Convergence analysis}

This section addresses the convergence properties of the self-tuning algorithm subject to both modeling errors in $\hat{\mathbf{P}}_{\mathbf{2}}$ and the presence of sensor noise $\boldsymbol{n}_{\mathbf{0}}$ and $\boldsymbol{n}_{\mathbf{1}}$. These properties are used to design the residual noise shaping filter $\mathbf{N}$ and to find an expression for $\mu$ in (34).

To compare the result of the self-tuning controller with the model-based controller from Section 4, the weight estimation error will be defined as $\Delta \boldsymbol{w}(k)=\boldsymbol{w}(k)-\boldsymbol{w}^{*}$ with $\boldsymbol{w}^{*}$ from (26). Subtracting $\boldsymbol{w}^{*}$ from both sides of (34) it is found that

$$
\Delta \boldsymbol{w}(k+1)=\Delta \boldsymbol{w}(k)-\mu(k)[\mathbf{N}(q) \tilde{\boldsymbol{\Psi}}(k)]^{T} \boldsymbol{e}(k) .
$$

Eq. (35) describes the development of $\Delta \boldsymbol{w}(k)$ over time, i.e. convergence and bias of the self-tuning filter with respect to the model-based controller. To analyze the asymptotic behavior of $\boldsymbol{\Delta} \boldsymbol{w}(k)$ with respect to the disturbances, (35) must be rewritten because $\boldsymbol{e}(k)$ is still a function of $\boldsymbol{\Delta} \boldsymbol{w}(k)$. Reconsider $\boldsymbol{e}(k)$ in (32), which contains $\boldsymbol{y}_{\mathbf{1}}(k)$ and note that without loss of generality $\boldsymbol{y}_{\mathbf{1}}$ can be written as

$$
\boldsymbol{y}_{\mathbf{1}}(k)=\underbrace{-H_{(\alpha, n)}(q) \mathbf{P}_{\mathbf{2}}(q) \boldsymbol{\Psi}(k) \boldsymbol{w}^{*}}_{\boldsymbol{y}_{\mathbf{2}}^{*}(k)}+\boldsymbol{\epsilon}_{\mathbf{0}}(k),
$$

with $\boldsymbol{y}_{\mathbf{2}}^{*}(k)$ representing the (noise-free) secondary path output in case $\boldsymbol{w}(k):=\boldsymbol{w}^{*}$, and $\boldsymbol{\epsilon}_{\mathbf{0}}(k) \in \mathbb{R}^{6}$ describing the residual error for which $\boldsymbol{y}_{\mathbf{2}}^{*}$ cannot compensate. In (36), 
the noise-free regression matrix $\boldsymbol{\Psi}$ is used rather than $\tilde{\boldsymbol{\Psi}}$ since $\boldsymbol{y}_{\mathbf{1}}$ is obtained from the noise-free input signal $\boldsymbol{a}_{\mathbf{0}}$, see Figure 7. Similar to the definition of $\tilde{\boldsymbol{\Psi}}$ in (26), $\boldsymbol{\Psi}$ is defined as

$$
\begin{gathered}
\boldsymbol{\Psi}(k)=\left[\begin{array}{ccc}
\boldsymbol{\psi}^{T}(k) & \ldots & \mathbf{0} \\
\vdots & \ddots & \vdots \\
\mathbf{0} & \ldots & \boldsymbol{\psi}^{T}(k)
\end{array}\right], \\
\boldsymbol{\psi}(k)=\left[\begin{array}{c}
\boldsymbol{a}_{\mathbf{0}}(k) \\
\beta H_{(\alpha, n)}(q) \boldsymbol{a}_{\mathbf{0}}(k)
\end{array}\right] .
\end{gathered}
$$

Substitution of (36) in (32) and after some rewriting it is found that

$$
\begin{gathered}
\boldsymbol{e}(k)=\left[\mathbf{N}(q) \hat{\mathbf{P}}_{\mathbf{2}}^{-1}(q) \mathbf{P}_{\mathbf{2}}(q) \tilde{\boldsymbol{\Psi}}(k)\right] \Delta \boldsymbol{w}(k) \\
+\mathbf{N}(q) \hat{\mathbf{P}}_{\mathbf{2}}^{-1}(q) \mathbf{P}_{\mathbf{2}}(q)(\tilde{\boldsymbol{\Psi}}(k)-\boldsymbol{\Psi}(k)) \boldsymbol{w}^{*} \\
+H_{(\alpha, n)}^{-1}(q) \mathbf{N}(q) \hat{\mathbf{P}}_{\mathbf{2}}^{-1}(q)\left(\boldsymbol{\epsilon}_{\mathbf{0}}(k)+\boldsymbol{n}_{\mathbf{1}}(k)\right) .
\end{gathered}
$$

Moreover, when (38) is substituted in (35) it is obtained that

$$
\begin{aligned}
\Delta \boldsymbol{w}(k+1)= & {\left[\mathbf{I}_{\mathbf{6}}-\mu(k) \mathbf{G}_{\mathbf{1}}(k)\right] \Delta \boldsymbol{w}(k) } \\
& +\mu(k) \mathbf{G}_{\mathbf{2}}(k)+\mu(k) \mathbf{G}_{\mathbf{3}}(k),
\end{aligned}
$$

with

$$
\begin{aligned}
& \mathbf{G}_{\mathbf{1}}(k)= {[\mathbf{N}(q) \tilde{\mathbf{\Psi}}(k)]^{T}\left[\mathbf{N}(q) \hat{\mathbf{P}}_{\mathbf{2}}^{-1}(q) \mathbf{P}_{\mathbf{2}}(q) \tilde{\boldsymbol{\Psi}}(k)\right], } \\
& \mathbf{G}_{\mathbf{2}}(k)=[\mathbf{N}(q) \tilde{\mathbf{\Psi}}(k)]^{T} {\left[\mathbf{N}(q) \hat{\mathbf{P}}_{\mathbf{2}}^{-1}(q) \mathbf{P}_{\mathbf{2}}(q)(\mathbf{\Psi}(k)-\tilde{\mathbf{\Psi}}(k))\right] \boldsymbol{w}^{*}, } \\
& \mathbf{G}_{\mathbf{3}}(k)=-[\mathbf{N}(q) \tilde{\mathbf{\Psi}}(k)]^{T} \\
& {\left[H_{(\alpha, n)}^{-1}(q) \mathbf{N}(q) \hat{\mathbf{P}}_{\mathbf{2}}^{-1}(q)\left(\boldsymbol{\epsilon}_{\mathbf{0}}(k)+\boldsymbol{n}_{\mathbf{1}}(k)\right)\right] }
\end{aligned}
$$

Eq. (39) describes the development of $\Delta \boldsymbol{w}(k)$ over time as a function of the disturbances $\boldsymbol{a}_{\mathbf{0}}, \boldsymbol{n}_{\mathbf{0}}$ (via $\left.\boldsymbol{\Psi}, \tilde{\boldsymbol{\Psi}}\right), \boldsymbol{n}_{\mathbf{1}}$ and $\boldsymbol{\epsilon}_{\mathbf{0}}$. Convergence of $\boldsymbol{\Delta} \boldsymbol{w}(k)$ in terms of mean values should be evaluated given the stochastic nature of these disturbances. In so doing, the average updating direction can be obtained by computing the expected value $\mathbb{E}[\Delta \boldsymbol{w}(k+1)]$. Using again the assumption that $\boldsymbol{w}(k)$ is adapted slowly with respect to $\tilde{\boldsymbol{\Psi}}(k)$, see also Appendix A, it is obtained that $\mathbb{E}\left[\mathbf{G}_{\mathbf{1}}(k) \Delta \boldsymbol{w}(k)\right]=\mathbb{E}\left[\mathbf{G}_{\mathbf{1}}(k)\right] \mathbb{E}[\Delta \boldsymbol{w}(k)]$. Under this assumption, it is found that

$$
\begin{array}{r}
\mathbb{E}[\Delta \boldsymbol{w}(k+1)]=\mathbb{E}\left[\mathbf{I}_{\mathbf{6}}-\mu(k) \mathbf{G}_{\mathbf{1}}(k)\right] \mathbb{E}[\Delta \boldsymbol{w}(k)] \\
+\mu(k) \mathbb{E}\left[\mathbf{G}_{\mathbf{2}}(k)\right]+\mu(k) \mathbb{E}\left[\mathbf{G}_{\mathbf{3}}(k)\right] .
\end{array}
$$

Note that (41) describes the development of the mean weight estimation error $\mathbb{E}[\Delta \boldsymbol{w}(k)]$ in terms of expected values. Consequently, this equation provides information 1) regarding stability of the discrete-time recursive algorithm (Section 5.3.1), 2) the speed of convergence (Section 5.3.2), and 3) the steady-state mean estimation error $\mathbb{E}[\Delta \boldsymbol{w}(\infty)]$ (Section 5.3.3).

\subsubsection{Stability analysis}

Basically (41) shows that $\Delta \boldsymbol{w}(k)$ converges in terms of mean values if and only if all eigenvalues $\lambda_{i}$ of the term $\mathbb{E}\left[\mathbf{I}_{\mathbf{6}}-\mu(k) \mathbf{G}_{\mathbf{1}}(k)\right]$ lie within the unit circle and under the assumption that $\mathbb{E}\left[\mathbf{G}_{\mathbf{2}}(k)\right]$ and $\mathbb{E}\left[\mathbf{G}_{\mathbf{3}}(k)\right]$ are uniformly bounded. Using (40) and assuming that $\mathbf{N}(q)=n(q) \mathbf{I}_{\mathbf{6}}$ with single-input single-output transfer function $n(q)$ such that the order in which $\mathbf{N}$ and $\hat{\mathbf{P}}_{\mathbf{2}}^{-1}(q) \mathbf{P}_{\mathbf{2}}(q)$ appear can be exchanged, it follows that $\mathbb{E}\left[\mathbf{G}_{\mathbf{1}}(k)\right]$ can be written as

$$
\begin{aligned}
\mathbb{E}\left[\mathbf{G}_{\mathbf{1}}(k)\right]=\mathbb{E}\left[[\mathbf{N}(q) \tilde{\boldsymbol{\Psi}}(k)]^{T} .\right. \\
\\
{\left.\left[\hat{\mathbf{P}}_{\mathbf{2}}^{-1}(q) \mathbf{P}_{\mathbf{2}}(q) \mathbf{N}(q) \tilde{\mathbf{\Psi}}(k)\right]\right] . }
\end{aligned}
$$

In the case of perfect estimation $\hat{\mathbf{P}}_{\mathbf{2}}:=\mathbf{P}_{\mathbf{2}},(42)$ reduces to the autocorrelation matrix of $\mathbf{N}(q) \tilde{\mathbf{\Psi}}(k)$ which is per definition positive definite (assuming that $\tilde{\boldsymbol{\Psi}}$ is persistently exciting). As such, there exists $\mu(k)>0$ for which all eigenvalues $\lambda_{i}$ of $\mathbb{E}\left[\mathbf{I}_{\mathbf{6}}-\mu(k) \mathbf{G}_{\mathbf{1}}(k)\right]$ lie within the unit circle, hence stability is guaranteed. When the model $\hat{\mathbf{P}}_{\mathbf{2}}$ is not perfect, a sufficient condition for stability is given by the strictly positive real (SPR) condition [33], or

$$
\left(\hat{\mathbf{P}}_{\mathbf{2}}^{-1}\left(e^{j \omega}\right) \mathbf{P}_{\mathbf{2}}\left(e^{j \omega}\right)\right)^{H}+\hat{\mathbf{P}}_{\mathbf{2}}^{-1}\left(e^{j \omega}\right) \mathbf{P}_{\mathbf{2}}\left(e^{j \omega}\right) \succ 0 .
$$

The derivation of this condition, which is based on the work of [33], is given in Appendix B.

Remark 2. Eq. (43) requires an accurate model for $\mathbf{P}_{\mathbf{2}}$ which is the transfer function from actuator forces to platform accelerations. This transfer function is generally easy to obtain from frequency-domain system identification methods as described in, e.g., [34]. Therefore, it might be expected that $\hat{\mathbf{P}}_{\mathbf{2}}$ is accurate over a broad frequency range. Nevertheless, it is very difficult to perfectly estimate $\mathbf{P}_{\mathbf{2}}$ by $\hat{\mathbf{P}}_{\mathbf{2}}$ for all frequencies such that (43) will not likely be satisfied in practice. However, this does not imply instability because (43) only gives a sufficient condition. Furthermore, the noise shaping filter can be used to suppress the destabilizing effect of frequencies for which (43) is not satisfied. Moreover, [8] explains that robust convergence can be improved by adding damping to resonance peaks using feedback control. Another method to stabilize convergence is to add plant regularization [8, 35] which means that the plant is augmented with virtual plant dynamics. This method improves convergence but generally leads to biased estimates of the parameters.

Provided that the eigenvalues of $\left[\mathbf{I}_{\mathbf{6}}-\mu(k) \mathbf{G}_{\mathbf{1}}(k)\right]$ lie within the unit circle, and assuming $\hat{\mathbf{P}}_{\mathbf{2}}:=\mathbf{P}_{\mathbf{2}}$ such that the eigenvalues of $\mathbf{G}_{\mathbf{1}}(k)$ are positive, lower and upper bounds for $\mu(k)$ are defined as

$$
0<\mu(k)<\frac{2}{\|\mathbf{N}(q) \tilde{\boldsymbol{\Psi}}(k)\|_{2}^{2}},
$$

explicitly using the fact that the maximum eigenvalue $\lambda_{\max }$ of $\mathbf{G}_{\mathbf{1}}$ is always smaller or equal to the maximum singular 
value of $\mathbf{G}_{\mathbf{1}}$ which is given by the 2 -norm of $\mathbf{G}_{\mathbf{1}}$. To satisfy (44) the adaptation rate is normalized, or

$$
\mu(k)=\frac{\bar{\mu}}{\epsilon+\|\mathbf{N}(q) \tilde{\mathbf{\Psi}}(k)\|_{2}^{2}},
$$

with $\epsilon>0$ a small positive regularization constant to provide an upper bound on $\mu(k)$, and $\bar{\mu} \in(0,2)$ the normalized adaptation rate. Using (45) the self-tuning algorithm becomes a special form of the normalized LMS algorithm [21] in which $\mu(k)$ is dependent on $\tilde{\boldsymbol{\Psi}}(k)$ which by itself is a function of $\tilde{\boldsymbol{a}}_{\mathbf{0}}$. As such, stability of the algorithm becomes independent of the power of $\tilde{\boldsymbol{a}}_{\mathbf{0}}$. On the one hand, $\bar{\mu}$ must be chosen small enough to prevent instability by violating the slow-adaptation assumption; the largest stabilizing step size may be very small if $\hat{\mathbf{P}}_{\mathbf{2}} \neq \mathbf{P}_{\mathbf{2}}$. Moreover, the steady-state variance on the parameters depends on the choice for $\bar{\mu}[21,36]$. To ensure that the estimation errors in the parameters are sufficiently small to obtain the desired transmissibility function, see Section 4.2.3, $\bar{\mu}$ must be sufficiently small. On the other hand, too slow adaptation rates will induce unnecessarily long convergence times, which is generally undesired.

\subsubsection{Convergence speed}

In Section 5.3.1 conditions on the eigenvalues $\lambda_{i}$ of $\left(\mathbf{I}_{\mathbf{6}}-\mu(k) \mathbf{G}_{\mathbf{1}}(k)\right)$ were given to achieve convergence of the self-tuning algorithm. In addition, to get uniform convergence speeds for all parameters in $\boldsymbol{w}(k)$, all eigenvalues $\lambda_{i}$ should be identical [9]. In this section, the convergence speed of the parameters is analyzed for the case $\hat{\mathbf{P}}_{\mathbf{2}}=\mathbf{P}_{\mathbf{2}}$. Furthermore, for simplicity, neither input sensor noise nor residual noise shaping will be considered, i.e. $\boldsymbol{n}_{\mathbf{0}}=\mathbf{0}$ and $\mathbf{N}=\mathbf{I}_{\mathbf{6}}$, and $\boldsymbol{a}_{\mathbf{0}}$ is assumed to be a ZMWN process, see Definition 3. Under these conditions, uniform convergence is obtained if

$$
\beta=\frac{1}{\left\|H_{(\alpha, n)}(q)\right\|_{2}},
$$

with $\left\|H_{(\alpha, n)}(q)\right\|_{2} \in \mathbb{R}$. The full derivation of (46) can be found in Appendix C.

Definition 3. Two uncorrelated zero-mean Gaussian white noise $(Z M W N)$ processes $\boldsymbol{u}_{\mathbf{1}}$ and $\boldsymbol{u}_{\mathbf{2}}$ have at every time sample $k \in \mathbb{Z}$ the properties that

$$
\begin{aligned}
& \mathbb{E}\left[\boldsymbol{u}_{\boldsymbol{j}}(k) \boldsymbol{u}_{\boldsymbol{j}}^{T}(k)\right]=\sigma_{u_{j}}^{2} \mathbf{I}_{\mathbf{6}}, \quad j \in\{1,2\}, \\
& \mathbb{E}\left[\boldsymbol{u}_{\boldsymbol{j}}(k) \boldsymbol{u}_{\boldsymbol{j}}^{T}(k-i)\right]=\mathbf{0} \forall i \in \mathbb{Z} \backslash\{0\}, \quad j \in\{1,2\}, \\
& \mathbb{E}\left[\boldsymbol{u}_{\mathbf{1}}(i) \boldsymbol{u}_{\mathbf{2}}(k)\right]=\mathbf{0} \forall i \in \mathbb{Z},
\end{aligned}
$$

with $\sigma_{u_{j}}^{2}$ representing the variance of the signals in $\boldsymbol{u}_{\boldsymbol{j}}$.

\subsubsection{Steady-state mean weight estimation error}

Having discussed the conditions for stability and convergence of the self-tuning algorithm, explicit expressions for the steady-state mean weight estimation error $\mathbb{E}[\Delta \boldsymbol{w}(\infty)]$, hence the bias in the estimates, can be derived. Bias will be related to the fact that the self-tuning filter aims at minimizing $\boldsymbol{e}^{T}(k) \boldsymbol{e}(k)$, while the model-based controller design is based on minimization of $\mathcal{T}$. The latter is posed in the frequency domain and obviously neglecting the contributions of sensor noise and unmodeled dynamics to $\boldsymbol{e}(k)$. As a result, bias will appear if $\mathbb{E}\left[\mathbf{G}_{\mathbf{2}}(k)\right]$ and $\mathbb{E}\left[\mathbf{G}_{\mathbf{3}}(k)\right]$ in (41) are non-zero. In Appendix D, the expression for bias is derived as

$$
\begin{aligned}
& \mathbb{E}[\Delta \boldsymbol{w}(\infty)]=-\frac{\sigma_{n_{0}}^{2}}{\sigma_{a_{0}}^{2}+\sigma_{n_{0}}^{2}} \boldsymbol{w}^{*}-\frac{1}{\sigma_{a_{0}}^{2}+\sigma_{n_{0}}^{2}} . \\
& \mathbb{E}\left[[\tilde{\boldsymbol{\Psi}}(k)]^{T}\left[H_{(\alpha, n)}^{-1}(q) \hat{\mathbf{P}}_{\mathbf{2}}^{-1}(q) \boldsymbol{\epsilon}_{\mathbf{0}}(k)\right]\right] .
\end{aligned}
$$

The first term in (47) describes the contribution of input sensor noise $\boldsymbol{n}_{\mathbf{0}}$. If $\boldsymbol{n}_{\mathbf{0}}$ and $\boldsymbol{a}_{\mathbf{0}}$ are colored signals, this bias may be decreased (but not avoided) by appropriate design of $\mathbf{N}$ (note that $\mathbf{N}=\mathbf{I}_{\mathbf{6}}$ in the foregoing analysis). Since $\tilde{\boldsymbol{a}}_{\mathbf{0}}=\boldsymbol{a}_{\mathbf{0}}+\boldsymbol{n}_{\mathbf{0}}$ is filtered by $\mathbf{N}$, it follows that $\mathbf{N}$ must be designed such that the frequencies where $\boldsymbol{n}_{\mathbf{0}}$ is dominant over $\boldsymbol{a}_{\mathbf{0}}$ induce appropriate filtering, while the frequencies where $\boldsymbol{a}_{\mathbf{0}}$ is dominant over $\boldsymbol{n}_{\mathbf{0}}$ remain unaffected.

The second term in (47) describes the contribution of process noise $\boldsymbol{\epsilon}_{\mathbf{0}}$. This term accounts for the effects of all dynamics the feedforward controller cannot compensate for, see (36). In practice, $\boldsymbol{\epsilon}_{\mathbf{0}} \neq \mathbf{0}$ because the pure integrators of the Wiener solution have been replaced by weak integrators in (23). As such, $\boldsymbol{\epsilon}_{\mathbf{0}}$ will possess low-frequency contents if $\boldsymbol{a}_{\mathbf{0}}$ contains frequencies $\omega<\alpha$. Furthermore, the feedforward control law $\tilde{\boldsymbol{\Psi}}(q) \boldsymbol{w}^{*}$ is based on the assumption that the hard mounts are massless and can be fully described by a stiffness and a damping matrix. This excludes the effect of structural dynamics of the suspension itself that may occur at higher frequencies. Therefore, it is quite natural to expect that $\boldsymbol{\epsilon}_{\mathbf{0}}$ has high-frequency contents too. Hence, the residual noise shaping filter $\mathbf{N}$ should be designed in view of filtering both low- and high-frequency contents from $\boldsymbol{e}$, such that the effect of $\boldsymbol{\epsilon}_{\mathbf{0}}$ in terms of bias remains sufficiently small.

Remark 4. The described convergence analysis only discusses convergence in terms of mean values, i.e. $\mathbb{E}[\Delta \boldsymbol{w}(k)]$. Convergence in terms of mean square values, i.e. the variance $\mathbb{E}\left[(\Delta \boldsymbol{w}(k))(\Delta \boldsymbol{w}(k))^{T}\right]$, is not discussed. It can be stated that $\boldsymbol{n}_{\mathbf{0}} \neq \mathbf{0}, \boldsymbol{n}_{\mathbf{1}} \neq \mathbf{0}, \boldsymbol{\epsilon}_{\mathbf{0}} \neq \mathbf{0}$ and $\hat{\mathbf{P}}_{\mathbf{2}} \neq \mathbf{P}_{\mathbf{2}}$ all contribute to a larger mean-square-error (MSE); also a larger value for $\bar{\mu}$ increases the MSE. For a detailed discussion on calculating the mean-square performance in case $\boldsymbol{n}_{\mathbf{1}} \neq \mathbf{0}$ the reader is referred to [24].

\subsection{Simulation results}

Three simulation studies without sensor noise $\left(\boldsymbol{n}_{\mathbf{0}}=\right.$ $\boldsymbol{n}_{\mathbf{1}}=\mathbf{0}$ ) have been performed to show convergence and to provide guidelines on how to choose the self-tuning controller parameters. Herein, different designs are used for $H_{(\alpha, n)}$ in (16), namely $n=1,3,5$, to show the effect of increasing $n$. The cut-off frequency $\alpha=2 \times 2 \pi \mathrm{rad} / \mathrm{s}$ 


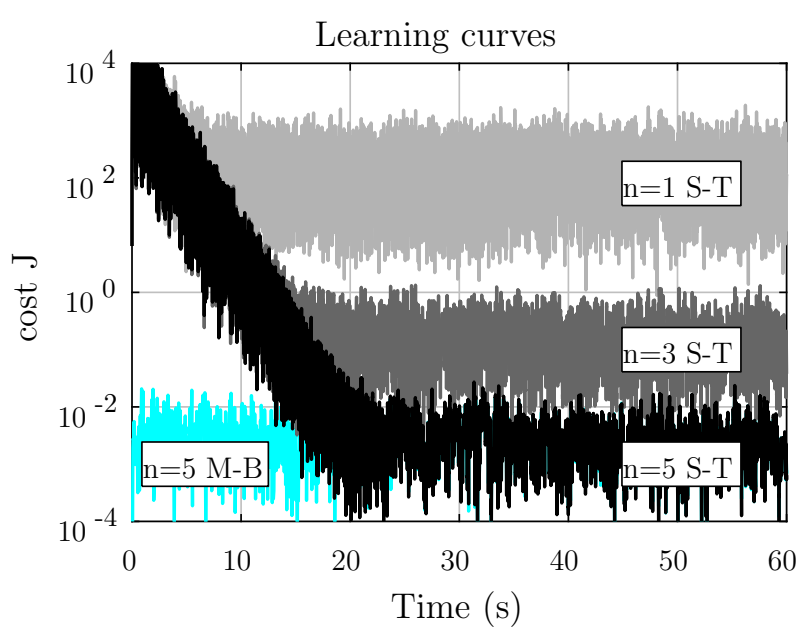

Figure 8: Learning Curves, showing convergence of the cost function defined in (29) when using self-tuning (S-T) controllers with $n^{\text {th }}$ order weak integrators and $n \in\{1,3,5\}$. For $n=5$, the plot shows that the cost of a self-tuning controller converges to the cost obtained in a simulation with model-based (M-B) control.

is chosen to be close to the lowest measurable frequency $(1 \mathrm{~Hz})$ of the sensors on the experimental setup to maximize performance. The primary and secondary path are obtained from the non-rigid-body model in (7). The feedback controller is given by (5). The inputs in $\boldsymbol{a}_{\mathbf{0}}$ satisfy Definition 3 with variance $\sigma_{a_{0}}^{2}=1$. The adaptation rate is set to $\bar{\mu}=10^{-3}$ which represents a tradeoff between stability, convergence time, and a sufficiently low variance on the steady-state estimates of the parameters. The constant $\epsilon=10^{-3}$ is $0.1 \%$ of the average input power to prevent division by zero. The sampling frequency $f_{s}=6400 \mathrm{~Hz}$ is set identical to that of the experimental setup. The parameter vector $\boldsymbol{w}(k)$ is initialized with zeros and is updated at every time sample $t_{k}$. In all simulation studies it is assumed that $\hat{\mathbf{P}}_{\mathbf{2}}=\mathbf{P}_{\mathbf{2}}$. The noise shaping filter is designed as

$$
\mathbf{N}(s)=\left(\frac{s}{s+5 \alpha}\right)^{5}\left(\frac{1000}{s+1000}\right)^{2} \mathbf{I}_{6}
$$

In view of the design rules derived at the end of Section 5.3.3, (48) contains a high-pass filter with a cut-off frequency of $5 \alpha \mathrm{rad} / \mathrm{s}$ to suppress low-frequency noise and a low-pass filter with two poles at $1000 \mathrm{rad} / \mathrm{s}$ to suppress the effects of unmodeled dynamics. Furthermore, the lowpass filter poles in $\mathbf{N}$ render $H_{(\alpha, n)}^{-1} \mathbf{N} \hat{\mathbf{P}}_{\mathbf{2}}^{-1}$ strictly proper to ensure a causal implementation in discrete-time.

Figure 8 shows the learning curves in terms of cost $J$ for the self-tuning controller. From the figure, it is clear that the controller parameters converge to minimum costs. It can also be observed that increasing $n$ in (16) leads to smaller residual costs, which is expected because $\boldsymbol{a}_{\mathbf{0}}$ is better rejected. Increasing $n$ leads to a longer convergence time to reach the accordingly smaller steady-state cost level, but even for $n=5$ the algorithm only needs 20 seconds to converge. Figure 9 shows the average relative bias of the parameters after convergence. It is observed

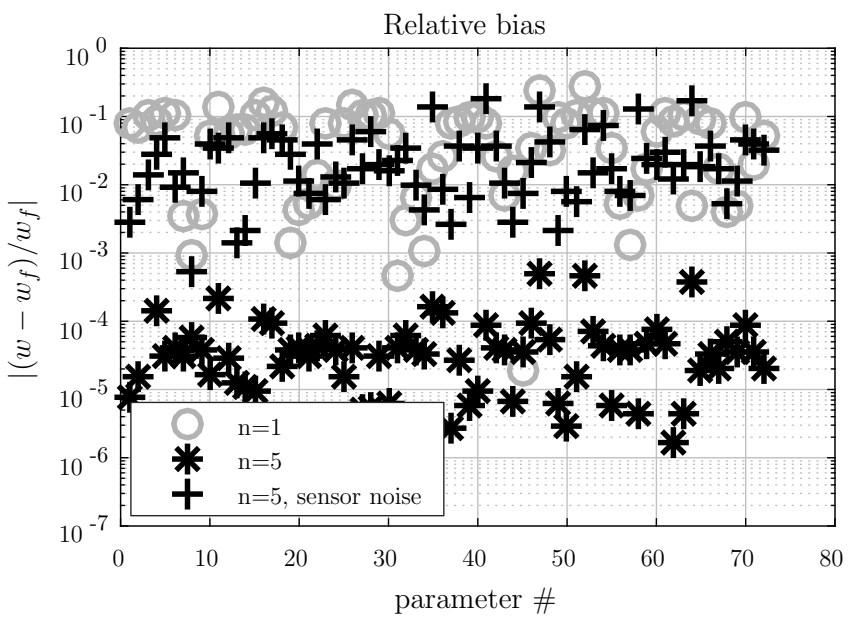

Figure 9: Average relative bias for the parameters after convergence for $n=1, n=5$ and $n=5$ with input sensor noise $\boldsymbol{n}_{\mathbf{0}}$. The figure shows that increasing $n$ leads to less bias.

that increasing $n$ leads to less bias, because the residual error in $\boldsymbol{\epsilon}_{\mathbf{0}}$ becomes smaller. For $n=5$, the average relative bias is less than $0.01 \%$, which implies that the parameters of the self-tuning controller converge to the actual damping and stiffness parameters of the system. The figure also shows the result of a fourth study with $n=5$ and additional sensor noise $\boldsymbol{n}_{\mathbf{0}}$ consisting of six uncorrelated ZMWN signals having variance $\sigma_{n_{0}}^{2}=0.01$. As expected from Section 5.3.3, the steady-state bias level increases to approximately $\frac{\sigma_{n_{0}}^{2}}{\sigma_{a_{0}}^{2}+\sigma_{n_{0}}^{2}} \approx 10^{-2}$.

Two important benefits of self-tuning feedforward control with respect to model-based control are derived from the bias plot in Figure 9. First, it is shown that the selftuning parameters converge to the actual system parameters with sufficient accuracy. This accuracy is said to be sufficient because for parameter uncertainties less than $1 \%$ the effect of sensor filtering is the dominant restriction on performance at most frequencies, see Figures 5 and 6 . It is important to note that, using self-tuning control, this convergence is reached without the need of doing an accurate (and costly) system identification procedure which would be necessary when using model-based control. Second, it is shown that bias appears in case of sensor noise, but this bias follows from minimization of the cost function in (29). In other words, the model-based controller is not the optimal solution for the noisy case, and this aspect is automatically compensated for in the self-tuning controller design.

\section{Experimental results}

The self-tuning feedforward controller from Section 5 is implemented on the experimental setup as discussed in Section 2 to validate the control strategy. Performance is evaluated in terms of the transmissibility matrix $\mathcal{T}$ in $z$-, 


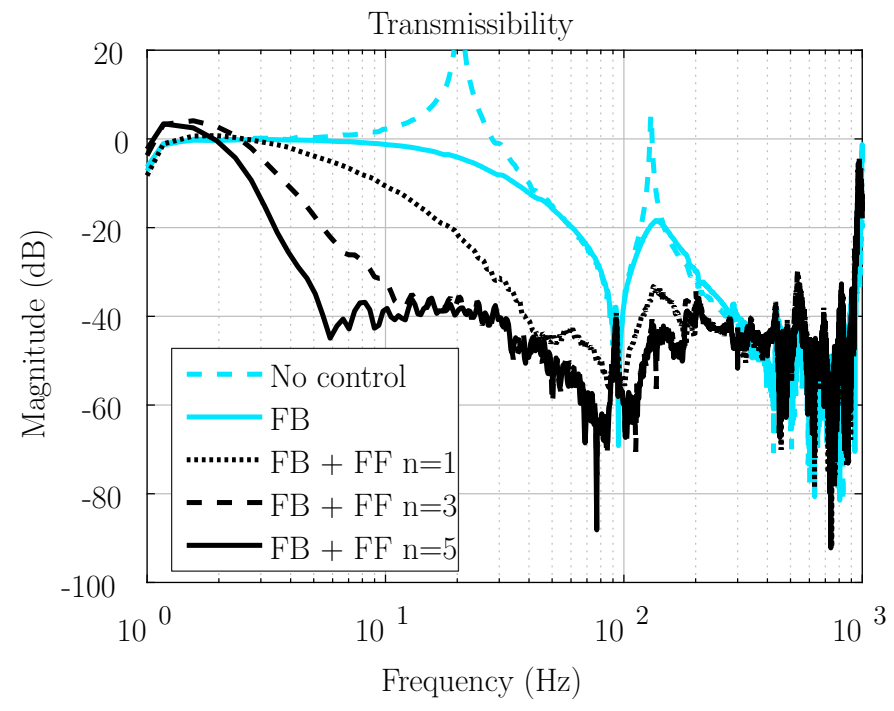

Figure 10: Measured transmissibilities in Z-direction. The passive and feedback controlled system response are compared with the system response including feedforward control. Notice that higher-order weak integrators in the feedforward controller result in a stronger roll-off for frequencies beyond $\alpha=2 \mathrm{~Hz}$.

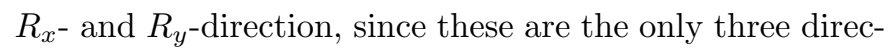
tions that can be excited using the vertical floor plate shakers, see Figure 1. Three uncorrelated Pseudo-RandomBinary-Sequence (PRBS) signals are provided to the floor plate shakers. A parametric model $\hat{\mathbf{P}}_{\mathbf{2}}$, needed for selftuning, is adopted from [27]. The self-tuning feedforward controller from Section 5 is implemented using $n=1,3,5$ and $\alpha=2 \mathrm{~Hz}$, together with the feedback controller from (5). After convergence of the parameters, the adaptation rate is set to $\bar{\mu}=0$ to make the system time-invariant such that the transmissibilities can be measured in terms of frequency response functions.

Measurement results of the control experiments are presented in Figures 10 and 11. Figure 10 shows the transmissibilities $\mathcal{T}$ in $z$-direction for the passive, the feedbackcontrolled, and the feedback- plus feedforward-controlled system. It is observed that increasing $n$ leads to more rolloff in the transmissibility as predicted in Figure 3. Figure 10 also shows that adding feedforward control leads to large performance improvements, because the transmissibility is decreased up to $40 \mathrm{~dB}$ with respect to the feedback-only controlled system for frequencies in the interval between 2 and $300 \mathrm{~Hz}$. The $40 \mathrm{~dB}$ saturation limit is similar to the limits predicted (and discussed) by sensor filtering in Figure 5. Therefore it is reasonable to assume that this saturation limit is dominantly caused by the effect of sensor filtering. This implies that the problems regarding parameter uncertainty in model-based control, see Figure 6, are sufficiently solved by applying self-tuning control. Furthermore, performance is limited due to the causal nature of the input disturbance measurement, i.e. the disturbance occurs prior to detection and compensation $[37,38,39]$. This causality argument results in the fact that active vibration isolation deteriorates performance at high frequencies due to a waterbed effect [38]. For frequencies below $2 \mathrm{~Hz}$, performance is slightly deteriorated due to application of the weak integrators, as discussed in Section 4. For frequencies beyond $400 \mathrm{~Hz}$ and at the $90 \mathrm{~Hz}$ anti-resonance frequency, sensor noise dominates the error response.

Figure 11 shows the MIMO measured transmissibilities $\mathcal{T}$ in $z, R_{x}$, and $R_{y}$ directions for the passive, the feedback-controlled and the feedback- plus feedforwardcontrolled case with $n=5$. The transmissibilities are obtained by three sequential experiments with different input signals for the floor plate shakers. The figure shows that the main diagonals of the transmissibility matrix associate with $40 \mathrm{~dB}$ reduction in all directions, similar to the performance of the measurements in Figure 10. The off-diagonal terms in the transmissibility matrix have different lower bounds because not all inputs and outputs have the same units (meters vs. radians).

\section{Conclusions}

This paper presents a feedforward control strategy for active vibration isolation of precision machinery. A modelbased feedforward controller to achieve perfect cancellation of floor vibrations is derived that only depends on the stiffness and damping properties of the suspension. To prevent problems with drift and actuator saturation, the feedforward controller gain is limited for frequencies below $2 \mathrm{~Hz}$ using higher-order weak integrators. Since pure model-based feedforward control is shown to be very sensitive for parameter estimation errors, the feedforward controller parameters are obtained by applying a self-tuning algorithm. Stability and minimization of bias in the parameter estimates are obtained by using a residual noise shaping filter. Simulations show that the parameters of the self-tuning controller converge to the actual system parameters with a predictable bias and in the presence of input sensor noise. An experimental validation of the self-tuning feedforward control strategy on an active hard-mounted vibration isolation system confirms the simulation results. Moreover, the validation shows that suppression of floor vibrations is improved up to $40 \mathrm{~dB}$ for frequencies between 2 and $300 \mathrm{~Hz}$ in the measured $3 \times 3$ MIMO transmissibility matrix.

\section{Acknowledgments}

This research was funded by the Impuls I research program from the Eindhoven University of Technology in collaboration with ASML. The authors appreciate the facilitation of the experimental setup by the Mechanical Automation and Mechatronics group at the University of Twente. 

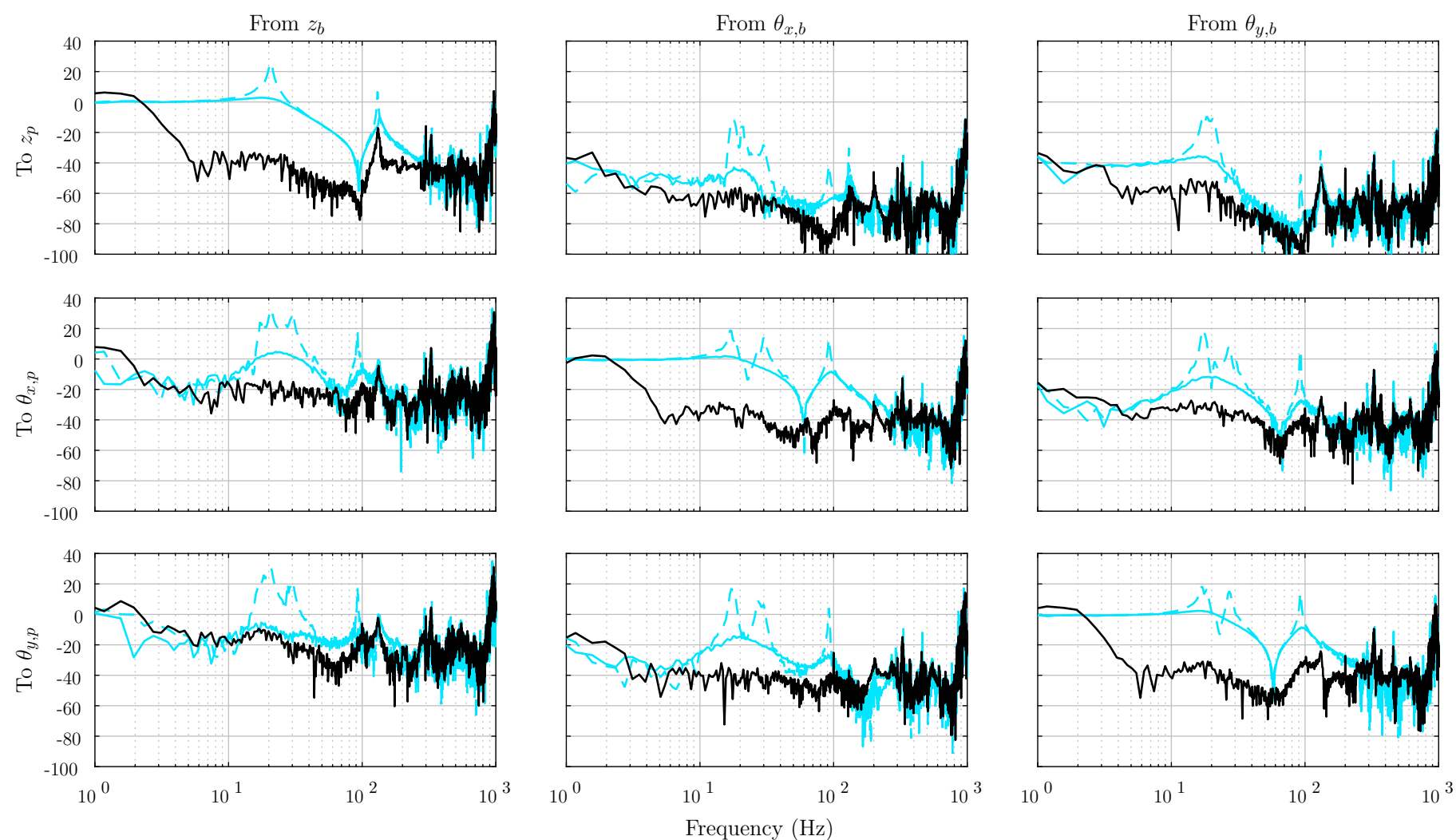

Figure 11: Measured transmissibilities for the MIMO system, for the cases passive (dashed blue), feedback-controlled (solid blue) and feedbackplus feedforward-controlled (black, using $n=5$ and $\alpha=2 \times 2 \pi \mathrm{rad} / \mathrm{s}$ ). Observe that all three plots on the main diagonal show up to $40 \mathrm{~dB}$ performance improvement, hence a natural extension of the results for single-axis control shown in Figure 10.

\section{Appendix A. Derivation of the gradient}

This Appendix derives a further expression for $\boldsymbol{e}(k)$ and the error gradient signal in (30). To this end, note from Figure 2 that $\tilde{\boldsymbol{a}}_{\mathbf{1}}$ can be defined as

$$
\tilde{\boldsymbol{a}}_{\mathbf{1}}(k)=\boldsymbol{y}_{\mathbf{1}}(k)+\mathbf{P}_{\mathbf{2}}(q) \boldsymbol{u}(k)+\boldsymbol{n}_{\mathbf{1}}(k) .
$$

Substitution of (A.1) in (31) and neglecting feedback con$\operatorname{trol}^{2}\left(\boldsymbol{u}_{\boldsymbol{F} \boldsymbol{B}}=\mathbf{0}\right)$ such that $\boldsymbol{u}=\boldsymbol{u}_{\boldsymbol{F F}}$ (see (27)) leads to

$$
\begin{aligned}
\boldsymbol{e}(k)= & H_{(\alpha, n)}^{-1}(q) \mathbf{N}(q) \hat{\mathbf{P}}_{\mathbf{2}}^{-1}(q) \boldsymbol{y}_{\mathbf{1}}(k) \\
& +\mathbf{N}(q) \hat{\mathbf{P}}_{\mathbf{2}}^{-1}(q) \mathbf{P}_{\mathbf{2}}(q)[\tilde{\boldsymbol{\Psi}}(k) \boldsymbol{w}(k)] \\
& +H_{(\alpha, n)}^{-1}(q) \mathbf{N}(q) \hat{\mathbf{P}}_{\mathbf{2}}^{-1}(q) \boldsymbol{n}_{\mathbf{1}}(k) .
\end{aligned}
$$

Eq. (A.2) is not an explicit function of $\boldsymbol{w}(k)$, since $\boldsymbol{w}(k)$ is influenced by the time-shifting filters $\mathbf{N}(q), \hat{\mathbf{P}}_{\mathbf{2}}^{-1}(q)$, and $\mathbf{P}_{\mathbf{2}}(q)$. Hence, no direct (explicit) expression for the gradient $\frac{\partial \boldsymbol{e}(k)}{\partial \boldsymbol{w}}$ results. However, if $\boldsymbol{w}(k)$ is assumed to vary slowly with respect to $\tilde{\boldsymbol{\Psi}}(k)$, then $\tilde{\boldsymbol{\Psi}}(k)$ and $\boldsymbol{w}(k)$ can be

\footnotetext{
${ }^{2}$ It can be shown that for the case with feedback control the same self-tuning algorithm is obtained, with the only difference that $\hat{\mathbf{P}}_{\mathbf{2}}$ in (31) must be replaced by the process sensitivity $\left(\mathbf{I}_{\mathbf{6}}+\right.$ $\left.\hat{\mathbf{P}}_{\mathbf{2}}(q) \mathbf{C}_{\mathbf{F B}}(q)\right)^{-1} \hat{\mathbf{P}}_{\mathbf{2}}(q)$
}

considered independent [33] such that

$$
\begin{aligned}
\boldsymbol{e}(k) \approx & H_{(\alpha, n)}^{-1}(q) \mathbf{N}(q) \hat{\mathbf{P}}_{\mathbf{2}}^{-1}(q) \boldsymbol{y}_{\mathbf{1}}(k) \\
& +\left[\mathbf{N}(q) \hat{\mathbf{P}}_{\mathbf{2}}^{-1}(q) \mathbf{P}_{\mathbf{2}}(q) \tilde{\mathbf{\Psi}}(k)\right] \boldsymbol{w}(k) \\
& +H_{(\alpha, n)}^{-1}(q) \mathbf{N}(q) \hat{\mathbf{P}}_{\mathbf{2}}^{-1}(q) \boldsymbol{n}_{\mathbf{1}}(k) .
\end{aligned}
$$

As a result, the derivative of (A.3) with respect to $\boldsymbol{w}$ becomes

$$
\begin{aligned}
\frac{\partial \boldsymbol{e}(k)}{\partial \boldsymbol{w}} & \approx \mathbf{N}(q) \hat{\mathbf{P}}_{\mathbf{2}}^{-1}(q) \mathbf{P}_{\mathbf{2}}(q) \tilde{\mathbf{\Psi}}(k) \\
& \approx \mathbf{N}(q) \tilde{\mathbf{\Psi}}(k) \text { if } \hat{\mathbf{P}}_{\mathbf{2}}(q)=\mathbf{P}_{\mathbf{2}}(q) .
\end{aligned}
$$

Using (A.4), the gradient in (30) can be estimated by

$$
\begin{array}{rl}
\left(\frac{\partial J(k)}{\partial \boldsymbol{w}}\right)^{T} \approx 2[\mathbf{N}(q) \tilde{\mathbf{\Psi}}(k)]^{T} & \boldsymbol{e}(k) \\
& \text { if } \hat{\mathbf{P}}_{\mathbf{2}}(q)=\mathbf{P}_{\mathbf{2}}(q) .
\end{array}
$$

\section{Appendix B. Derivation of uncertainty bound for convergence}

In this appendix a sufficient condition is derived for convergence of the self-tuning algorithm presented in Section 5. The derivation is inspired by the work of Wang and Ren [33]. 
The derivation starts with (41), which shows that convergence in terms of mean values is obtained if the eigenvalues $\lambda_{i}$ of $\mathbb{E}\left[\mathbf{I}_{\mathbf{6}}-\mu(k) \mathbf{G}_{\mathbf{1}}(k)\right]$ lie within the unit circle. Provided that all eigenvalues of $\mathbf{A}=\mathbb{E}\left[\mathbf{G}_{\mathbf{1}}(k)\right]$ have positive real parts, it follows that a positive step-size $\mu(k)$ can be found such that all $\left|\lambda_{i}\right|<1$. According to (42) this matrix is given by

$$
\mathbf{A}=\mathbb{E}\left[\left[\boldsymbol{\Psi}_{\mathbf{N}}(k)\right]^{T}\left[\hat{\mathbf{P}}_{\mathbf{2}}^{-1}(q) \mathbf{P}_{\mathbf{2}}(q) \mathbf{\Psi}_{\mathbf{N}}(k)\right]\right]
$$

with $\boldsymbol{\Psi}_{\mathbf{N}}(k)=\mathbf{N}(q) \tilde{\boldsymbol{\Psi}}(k)$. Next, define a random vector $\boldsymbol{L} \in \mathbb{R}^{72}$ and compute

$$
\begin{aligned}
& \boldsymbol{L}^{T}(\mathbf{A}+\left.\mathbf{A}^{T}\right) \boldsymbol{L}=\mathbb{E}\left[[\boldsymbol{\Xi}(k)]^{T}\left[\hat{\mathbf{P}}_{\mathbf{2}}^{-1}(q) \mathbf{P}_{\mathbf{2}}(q) \boldsymbol{\Xi}(k)\right]\right] \\
&+\mathbb{E}\left[\left[\hat{\mathbf{P}}_{\mathbf{2}}^{-1}(q) \mathbf{P}_{\mathbf{2}}(q) \boldsymbol{\Xi}(k)\right]^{T}[\boldsymbol{\Xi}(k)]\right]
\end{aligned}
$$

with

$$
\boldsymbol{\Xi}(k)=\boldsymbol{\Psi}_{\mathbf{N}}(k) \boldsymbol{L} .
$$

The output of (B.2) is scalar valued. Using the property $\mathbf{u}_{\mathbf{1}}^{\mathbf{T}} \mathbf{u}_{\mathbf{2}}=\operatorname{tr}\left\{\mathbf{u}_{\mathbf{1}} \mathbf{u}_{\mathbf{2}}^{\mathbf{T}}\right\}$, it follows that

$$
\begin{aligned}
& \boldsymbol{L}^{T}(\mathbf{A}\left.+\mathbf{A}^{T}\right) \boldsymbol{L}=\operatorname{tr}\left\{\mathbb{E}\left[\boldsymbol{\Xi}(k)\left[\hat{\mathbf{P}}_{\mathbf{2}}^{-1}(q) \mathbf{P}_{\mathbf{2}}(q) \boldsymbol{\Xi}(k)\right]^{T}\right]\right. \\
&\left.+\mathbb{E}\left[\left[\hat{\mathbf{P}}_{\mathbf{2}}^{-1}(q) \mathbf{P}_{\mathbf{2}}(q) \boldsymbol{\Xi}(k)\right][\boldsymbol{\Xi}(k)]^{T}\right]\right\} .
\end{aligned}
$$

Next, Parseval's theorem is used to convert (B.4) to the frequency domain, or

$$
\begin{aligned}
& \boldsymbol{L}^{T}\left(\mathbf{A}+\mathbf{A}^{T}\right) \boldsymbol{L}=\frac{1}{2 \pi} \int_{\pi}^{\pi} \operatorname{tr}\left\{\mathbf{S}_{\boldsymbol{\Xi}}\left(e^{j \omega}\right)\right. \\
& \left.\left[\left(\hat{\mathbf{P}}_{\mathbf{2}}^{-1}\left(e^{j \omega}\right) \mathbf{P}_{\mathbf{2}}\left(e^{j \omega}\right)\right)^{H}+\hat{\mathbf{P}}_{\mathbf{2}}^{-1}\left(e^{j \omega}\right) \mathbf{P}_{\mathbf{2}}\left(e^{j \omega}\right)\right]\right\} \mathrm{d} \omega
\end{aligned}
$$

with $\mathbf{S}_{\boldsymbol{\Xi}}\left(e^{j \omega}\right)$ representing the power spectrum matrix of $\boldsymbol{\Xi}(k)$. In (B.5) the cyclic property for the trace operator, or $\operatorname{tr}\{A B C\}=\operatorname{tr}\{B C A\}$, is used to isolate $\mathbf{S}_{\boldsymbol{\Xi}}$. Observe that the integrand of (B.5) is the trace of two matrices. The matrix $\mathbf{S}_{\boldsymbol{\Xi}}$ represents a power spectrum matrix which is per definition positive definite if the input is sufficiently exciting. If now the strictly positive real (SPR) condition [33] is used, or

$$
\left(\hat{\mathbf{P}}_{\mathbf{2}}^{-1}\left(e^{j \omega}\right) \mathbf{P}_{\mathbf{2}}\left(e^{j \omega}\right)\right)^{H}+\hat{\mathbf{P}}_{\mathbf{2}}^{-1}\left(e^{j \omega}\right) \mathbf{P}_{\mathbf{2}}\left(e^{j \omega}\right) \succ 0,
$$

(where $\mathbf{M} \succ 0$ means that the Hermitian matrix $\mathbf{M}$ is positive definite, i.e., all of its eigenvalues are greater than zero) then the trace contains the product of two symmetric positive definite matrices (under the assumption that the input is sufficiently exciting such that $\mathbf{S}_{\boldsymbol{\Xi}} \succ 0$ ). Using (B.6), it follows that

$$
\boldsymbol{L}^{T}\left(\mathbf{A}+\mathbf{A}^{T}\right) \boldsymbol{L}>0 \quad \text { for } \boldsymbol{L} \neq \mathbf{0}
$$

Now, reconsider the mean weight update equation in (41). Define $\mathbb{E}[\Delta \boldsymbol{w}(k)]=\boldsymbol{x}(k)$ and assume there is no sensor noise and process noise such that $\mathbf{G}_{\mathbf{2}}(k)=\mathbf{G}_{\mathbf{3}}(k)=\mathbf{0}$. Then, (41) reduces to

$$
\boldsymbol{x}(k+1)=[\mathbf{I}-\mu \mathbf{A}] \boldsymbol{x}(k)
$$

Next, a Lyapunov function $V(k)=\boldsymbol{x}^{T}(k) \boldsymbol{x}(k) \geq 0$ is defined, with

$$
\begin{aligned}
\Delta V(k) & =V(k+1)-V(k) \\
& =\mu^{2} \boldsymbol{x}^{T} \mathbf{A}^{T} \mathbf{A} \boldsymbol{x}-\mu \boldsymbol{x}^{T}\left(\mathbf{A}+\mathbf{A}^{T}\right) \boldsymbol{x} .
\end{aligned}
$$

Since $\boldsymbol{x}^{T} \mathbf{A}^{T} \mathbf{A} \boldsymbol{x}>0$ because of its quadratic form, and $\boldsymbol{x}^{T}\left(\mathbf{A}+\mathbf{A}^{T}\right) \boldsymbol{x}>0$ because of (B.7), it holds that there always exists a sufficiently small $\mu$ such that $\mu^{2} \boldsymbol{x}^{T} \mathbf{A}^{T} \mathbf{A} \boldsymbol{x}<\mu \boldsymbol{x}^{T}\left(\mathbf{A}+\mathbf{A}^{T}\right) \boldsymbol{x}$. Then, it follows from combining (B.9) and (B.7), and for sufficiently small $\mu$, that $\Delta V(k)<0$ which implies that the self-tuning algorithm converges.

\section{Appendix C. Derivation of expected values and optimal convergence speed}

This appendix shows that for the conditions $\hat{\mathbf{P}}_{\mathbf{2}}=\mathbf{P}_{\mathbf{2}}$, $\mathbf{N}=\mathbf{I}_{\mathbf{6}}, \beta=\frac{1}{\left\|H_{(\alpha, n)}(q)\right\|_{2}}$ with $\left\|H_{(\alpha, n)}(q)\right\|_{2} \in \mathbb{R}$, and $\boldsymbol{a}_{\mathbf{0}}, \boldsymbol{n}_{\mathbf{0}}, \boldsymbol{n}_{\mathbf{1}}$ are ZMWN processes satisfying the properties from Definition 3, the following two properties hold: (1) $\mathbb{E}\left[\mathbf{G}_{\mathbf{1}}(k)\right]=\left(\sigma_{a_{0}}^{2}+\sigma_{n_{0}}^{2}\right) \mathbf{I}_{\mathbf{7 2}}$, and (2) uniform convergence speed of the parameters $\boldsymbol{w}(k)$ is obtained in terms of mean values.

Under the given assumptions, $\mathbb{E}\left[\mathbf{G}_{\mathbf{1}}(k)\right]$ in (41) reduces to

$$
\begin{aligned}
& \mathbb{E}\left[\mathbf{G}_{\mathbf{1}}(k)\right]=\mathbb{E}\left[\tilde{\boldsymbol{\Psi}}^{T}(k) \tilde{\boldsymbol{\Psi}}(k)\right] \\
& =\mathbb{E}\left[\begin{array}{ccc}
\tilde{\boldsymbol{\psi}}(k) \tilde{\boldsymbol{\psi}}^{T}(k) & \ldots & \mathbf{0} \\
\vdots & \ddots & \vdots \\
\mathbf{0} & \ldots & \tilde{\boldsymbol{\psi}}(k) \tilde{\boldsymbol{\psi}}^{T}(k)
\end{array}\right]
\end{aligned}
$$

Because $\mathbb{E}\left[\tilde{\boldsymbol{\Psi}}^{T}(k) \tilde{\boldsymbol{\Psi}}(k)\right]$ is block-diagonal, the eigenvalues of $\mathbb{E}\left[\tilde{\boldsymbol{\Psi}}^{T}(k) \tilde{\boldsymbol{\Psi}}(k)\right]$ are determined by the eigenvalues of $\mathbb{E}\left[\tilde{\boldsymbol{\psi}}(k) \tilde{\boldsymbol{\psi}}^{T}(k)\right]$. From the definition of $\tilde{\boldsymbol{\psi}}(k)$ in $(37)$, the expression in (C.1) is derived. Assuming that $\tilde{\boldsymbol{a}}_{\mathbf{0}}=\boldsymbol{a}_{\mathbf{0}}+\boldsymbol{n}_{\mathbf{0}}$ consists of uncorrelated ZMWN signals $\boldsymbol{a}_{\mathbf{0}}, \boldsymbol{n}_{\mathbf{0}}$, it follows that the upper left part of (C.1) may be written as

$$
\mathbb{E}\left[\tilde{\boldsymbol{a}}_{\mathbf{0}}(k) \tilde{\boldsymbol{a}}_{\mathbf{0}}^{T}(k)\right]=\left(\sigma_{a_{0}}^{2}+\sigma_{n_{0}}^{2}\right) \mathbf{I}_{\mathbf{6}}
$$

If the discrete-time weak integrator $H_{(\alpha, n)}(q)$ is derived by discretization of (16) using the zero-order-hold method, the relative degree of $H_{(\alpha, n)}(q)$ becomes one, which implies that there is no direct feedthrough term in the output equation of the filter. As a result, $H_{(\alpha, n)}(q) \boldsymbol{a}_{\mathbf{0}}(k)$ can be expressed as a function of $\tilde{\boldsymbol{a}}_{\mathbf{0}}(k-1), \ldots, \tilde{\boldsymbol{a}}_{\mathbf{0}}(k-n)$. Given 


$$
\mathbb{E}\left[\tilde{\boldsymbol{\psi}}(k) \tilde{\boldsymbol{\psi}}^{T}(k)\right]=\mathbb{E}\left[\begin{array}{c:c}
\tilde{\boldsymbol{a}}_{\mathbf{0}}(k) \tilde{\boldsymbol{a}}_{\mathbf{0}}^{T}(k) & \tilde{\boldsymbol{a}}_{\mathbf{0}}(k)\left[\beta H_{(\alpha, n)}(q) \tilde{\boldsymbol{a}}_{\mathbf{0}}(k)\right]^{T} \\
\hdashline\left[\beta H_{(\alpha, n)}(q) \tilde{\boldsymbol{a}}_{\mathbf{0}}(k)\right] & \tilde{\boldsymbol{a}}_{\mathbf{0}}^{T}(k) \\
{\left[\beta H_{(\alpha, n)}(q) \tilde{\boldsymbol{a}}_{\mathbf{0}}(k)\right]\left[\beta H_{(\alpha, n)}(q) \tilde{\boldsymbol{a}}_{\mathbf{0}}(k)\right]^{T-}}
\end{array}\right] .
$$

Definition 3, it follows that $\mathbb{E}\left[\tilde{\boldsymbol{a}}_{\mathbf{0}}(k) \tilde{\boldsymbol{a}}_{\mathbf{0}}^{T}(k-i)\right]=\mathbf{0}$ for $i=1,2, \ldots$, and therefore

$$
\mathbb{E}\left[\tilde{\boldsymbol{a}}_{\mathbf{0}}(k)\left[H_{(\alpha, n)}(q) \tilde{\boldsymbol{a}}_{\mathbf{0}}(k)\right]^{T}\right]=\mathbf{0},
$$

such that the upper right and lower left parts of (C.1) are zero too. Finally, an expression for the lower right part of the matrix in (C.1) can be obtained using Parseval's theorem, or

$$
\begin{aligned}
& \mathbb{E}\left[\left[\beta H_{(\alpha, n)}(q) \tilde{\boldsymbol{a}}_{\mathbf{0}}(k)\right]\left[\beta H_{(\alpha, n)}(q) \tilde{\boldsymbol{a}}_{\mathbf{0}}(k)\right]^{T}\right] \\
& =\frac{1}{2 \pi} \int_{-\pi}^{\pi} \beta^{2}\left(H_{(\alpha, n)}\left(e^{j \omega}\right)\left(\sigma_{a_{0}}^{2}+\sigma_{n_{0}}^{2}\right) \mathbf{I}_{\mathbf{6}} H_{(\alpha, n)}^{*}\left(e^{j \omega}\right)\right) \mathrm{d} \omega \\
& =\frac{\beta^{2}\left(\sigma_{a_{0}}^{2}+\sigma_{n_{0}}^{2}\right)}{2 \pi} \mathbf{I}_{\mathbf{6}} \int_{-\pi}^{\pi}\left(H_{(\alpha, n)}\left(e^{j \omega}\right) H_{(\alpha, n)}^{*}\left(e^{j \omega}\right)\right) \mathrm{d} \omega \\
& =\beta^{2}\left(\sigma_{a_{0}}^{2}+\sigma_{n_{0}}^{2} \mathbf{I}_{\mathbf{6}}\left\|H_{(\alpha, n)}(q)\right\|_{2}^{2} .\right.
\end{aligned}
$$

With $\beta=\frac{1}{\left\|H_{(\alpha, n)}(q)\right\|_{2}}$ it follows that

$$
\begin{aligned}
& \mathbb{E}\left[\left[\beta H_{(\alpha, n)}(q) \tilde{\boldsymbol{a}}_{\mathbf{0}}(k)\right]\left[\beta H_{(\alpha, n)}(q) \tilde{\boldsymbol{a}}_{\mathbf{0}}(k)\right]^{T}\right] \\
& \quad=\left(\sigma_{a_{0}}^{2}+\sigma_{n_{0}}^{2}\right) \mathbf{I}_{\mathbf{6}},
\end{aligned}
$$

Combining the results of (C.3), (C.4) and (C.5) it is found that

$$
\mathbb{E}\left[\tilde{\boldsymbol{\psi}}(k) \tilde{\boldsymbol{\psi}}^{T}(k)\right]=\left(\sigma_{a_{0}}^{2}+\sigma_{n_{0}}^{2}\right) \mathbf{I}_{\mathbf{1 2}},
$$

hence all eigenvalues of $\mathbb{E}\left[\tilde{\boldsymbol{\psi}}(k) \tilde{\boldsymbol{\psi}}^{T}(k)\right]$ equal $\left(\sigma_{a_{0}}^{2}+\sigma_{n_{0}}^{2}\right)$, which implies uniform convergence speed for all parameters. Furthermore, if the result of (C.6) is used in (C.2), it follows that $\mathbb{E}\left[\mathbf{G}_{\mathbf{1}}(k)\right]=\left(\sigma_{a_{0}}^{2}+\sigma_{n_{0}}^{2}\right) \mathbf{I}_{\mathbf{7 2}}$.

\section{Appendix D. Derivation of the bias}

An expression for the bias is obtained as follows. Assuming the self-tuning algorithm is stable (Section 5.3.1), it holds that $\mathbb{E}[\Delta \boldsymbol{w}(k+1)] \rightarrow \mathbb{E}[\Delta \boldsymbol{w}(k)]$ as $k \rightarrow \infty$. Then, from (41) it can be seen that

$$
\mathbb{E}[\Delta \boldsymbol{w}(\infty)]=\mathbb{E}\left[\mathbf{G}_{\mathbf{1}}(\infty)\right]^{-1}\left(\mathbb{E}\left[\mathbf{G}_{\mathbf{2}}(\infty)\right]+\mathbb{E}\left[\mathbf{G}_{\mathbf{3}}(\infty)\right]\right) .
$$

For the simplified case $\mathbf{N}=\mathbf{I}_{\mathbf{6}}, \hat{\mathbf{P}}_{\mathbf{2}}=\mathbf{P}_{\mathbf{2}}$, setting $\beta=$ $\frac{1}{\left\|H_{(\alpha, n)}(q)\right\|_{2}}$ as proposed in Section 5.3.2, and assuming that $\boldsymbol{a}_{\mathbf{0}}, \boldsymbol{n}_{\mathbf{0}}$, and $\boldsymbol{n}_{\mathbf{1}}$ are uncorrelated ZMWN processes satisfying the properties from Definition 3, the terms in (D.1) are given by

$$
\mathbb{E}\left[\mathbf{G}_{\mathbf{1}}(\infty)\right]=\mathbb{E}\left[\tilde{\mathbf{\Psi}}^{T}(k) \tilde{\mathbf{\Psi}}(k)\right]=\left(\sigma_{a_{0}}^{2}+\sigma_{n_{0}}^{2}\right) \mathbf{I}_{\mathbf{7 2}},
$$

$$
\begin{aligned}
\mathbb{E}\left[\mathbf{G}_{\mathbf{2}}(\infty)\right] & =\mathbb{E}\left[\tilde{\mathbf{\Psi}}^{T}(k)(\boldsymbol{\Psi}(k)-\tilde{\mathbf{\Psi}}(k))\right] \boldsymbol{w}^{*} \\
& =-\sigma_{n_{0}}^{2} \boldsymbol{w}^{*}, \\
\mathbb{E}\left[\mathbf{G}_{\mathbf{3}}(\infty)\right] & =-\mathbb{E}\left[[\tilde{\mathbf{\Psi}}(k)]^{T}\left[H_{(\alpha, n)}^{-1}(q) \hat{\mathbf{P}}_{\mathbf{2}}^{-1}(q) \boldsymbol{\epsilon}_{\mathbf{0}}(k)\right]\right] .
\end{aligned}
$$

The derivations regarding (D.2) and (D.3) are given in Appendix C. Eq. (D.4) is obtained from (40) as follows. Given $\tilde{\boldsymbol{a}}_{\mathbf{0}}=\boldsymbol{a}_{\mathbf{0}}+\boldsymbol{n}_{\mathbf{0}}$ it follows that $\tilde{\boldsymbol{\Psi}}(k)$ is affine in $\boldsymbol{n}_{\mathbf{0}}, \boldsymbol{a}_{\mathbf{0}}$. Moreover, the term $\left[H_{(\alpha, n)}^{-1}(q) \mathbf{N}(q) \hat{\mathbf{P}}_{\mathbf{2}}^{-1}(q) \boldsymbol{n}_{\mathbf{1}}(k)\right]$ is affine in $\boldsymbol{n}_{\mathbf{1}}$. Since $\boldsymbol{n}_{\mathbf{1}}$ is assumed to be uncorrelated with $\boldsymbol{a}_{\mathbf{0}}, \boldsymbol{n}_{\mathbf{0}}$, the term $\mathbb{E}\left[\mathbf{G}_{\mathbf{3}}(k)\right]$ no longer depends on $\boldsymbol{n}_{\mathbf{1}}$, thus $\boldsymbol{n}_{\mathbf{1}}$ will not contribute to bias.

Substitution of (D.2), (D.3), and (D.4) in (D.1) gives an expression for the bias in the parameter estimates, or

$$
\begin{gathered}
\mathbb{E}[\Delta \boldsymbol{w}(\infty)]=-\frac{\sigma_{n_{0}}^{2}}{\sigma_{a_{0}}^{2}+\sigma_{n_{0}}^{2}} \boldsymbol{w}^{*}-\frac{1}{\sigma_{a_{0}}^{2}+\sigma_{n_{0}}^{2}} . \\
\mathbb{E}\left[[\tilde{\boldsymbol{\Psi}}(k)]^{T}\left[H_{(\alpha, n)}^{-1}(q) \hat{\mathbf{P}}_{\mathbf{2}}^{-1}(q) \boldsymbol{\epsilon}_{\mathbf{0}}(k)\right]\right] .
\end{gathered}
$$




\section{Appendix E. Numerical values for the model}

$\mathbf{M}_{\mathbf{f}}=\left[\begin{array}{cccccccccccc}9.05 & 0 & 0 & 0 & 0.49 & 0 & -2.82 & 12.1 & -10.07 & -7.25 & 12.1 & 12.1 \\ 0 & 9.05 & 0 & -0.49 & 0 & 0 & 2.82 & 12.1 & -10.07 & -7.25 & -12.1 & -12.1 \\ 0 & 0 & 9.05 & 0 & 0 & 0 & 0 & 0 & 0 & 0 & 14.24 & 10.25 \\ 0 & -0.49 & 0 & 0.17 & 0 & 0 & -0.4 & -2.14 & 1.43 & 1.03 & 2.14 & 2.14 \\ 0.49 & 0 & 0 & 0 & 0.17 & 0 & -0.4 & 2.14 & -1.43 & -1.03 & 2.14 & 2.14 \\ 0 & 0 & 0 & 0 & 0 & 0.15 & 0 & 0 & 0.62 & 0.62 & 0 & 0 \\ -2.82 & 2.82 & 0 & -0.4 & -0.4 & 0 & 3.99 & 0 & 0 & 0 & -17.11 & -17.11 \\ 12.1 & 12.1 & 0 & -2.14 & 2.14 & 0 & 0 & 95.06 & -61.11 & -44 & 0 & 0 \\ -10.07 & -10.07 & 0 & 1.43 & -1.43 & 0.62 & 0 & -61.11 & 73.15 & 58.91 & 0 & 0 \\ -7.25 & -7.25 & 0 & 1.03 & -1.03 & 0.62 & 0 & -44 & 58.91 & 48.66 & 0 & 0 \\ 12.1 & -12.1 & 14.24 & 2.14 & 2.14 & 0 & -17.11 & 0 & 0 & 0 & 145.92 & 131.68 \\ 12.1 & -12.1 & 10.25 & 2.14 & 2.14 & 0 & -17.11 & 0 & 0 & 0 & 131.68 & 121.43\end{array}\right]$

$\mathbf{D}_{\mathbf{f}}=\left[\begin{array}{cccccccccccc}104.03 & 0 & 0 & 0 & -0.85 & 0 & 0 & 0 & 0 & 0 & 0 & 0 \\ 0 & 104.03 & 0 & 0.85 & 0 & 0 & 0 & 0 & 0 & 0 & 0 & 0 \\ 0 & 0 & 105.07 & 0 & 0 & 0 & 0 & 0 & 0 & 0 & 0 & 0 \\ 0 & 0.85 & 0 & 2.1 & 0 & 0 & 0 & 0 & 0 & 0 & 0 & 0 \\ -0.85 & 0 & 0 & 0 & 2.1 & 0 & 0 & 0 & 0 & 0 & 0 & 0 \\ 0 & 0 & 0 & 0 & 0 & 8.32 & 0 & 0 & 0 & 0 & 0 & 0 \\ 0 & 0 & 0 & 0 & 0 & 0 & 9551 & 0 & 0 & 0 & 0 & 0 \\ 0 & 0 & 0 & 0 & 0 & 0 & 0 & 1414 & 0 & 0 & 0 & 0 \\ 0 & 0 & 0 & 0 & 0 & 0 & 0 & 0 & 166591 & 132478 & 0 & 0 \\ 0 & 0 & 0 & 0 & 0 & 0 & 0 & 0 & 132478 & 107916 & 0 & 0 \\ 0 & 0 & 0 & 0 & 0 & 0 & 0 & 0 & 0 & 0 & 5163 & 4113 \\ 0 & 0 & 0 & 0 & 0 & 0 & 0 & 0 & 0 & 0 & 4113 & 3358\end{array}\right]$

$\mathbf{K}_{\mathbf{f}}=\left[\begin{array}{ccccccccccccc}117800 & 0 & 0 & 0 & 0 & 0 & 0 & 0 & 0 & 0 & 0 & 0 \\ 0 & 117800 & 0 & 0 & 0 & 0 & 0 & 0 & 0 & 0 & 0 & 0 \\ 0 & 0 & 169794 & 0 & 0 & 0 & 0 & 0 & 0 & 0 & 0 & 0 \\ 0 & 0 & 0 & 3279 & 0 & 0 & 0 & 0 & 0 & 0 & 0 & 0 \\ 0 & 0 & 0 & 0 & 3279 & 0 & 0 & 0 & 0 & 0 & 0 & 0 \\ 0 & 0 & 0 & 0 & 0 & 8685 & 0 & 0 & 0 & 0 & 0 & 0 \\ 0 & 0 & 0 & 0 & 0 & 0 & 4.78 \cdot 10^{8} & 0 & 0 & 0 & 0 & 0 \\ 0 & 0 & 0 & 0 & 0 & 0 & 0 & 7.07 \cdot 10^{7} & 0 & 0 & 0 & 0 \\ 0 & 0 & 0 & 0 & 0 & 0 & 0 & 0 & 8.33 \cdot 10^{9} & 6.62 \cdot 10^{9} & 0 & 0 \\ 0 & 0 & 0 & 0 & 0 & 0 & 0 & 0 & 6.62 \cdot 10^{9} & 5.40 \cdot 10^{9} & 0 & 0 \\ 0 & 0 & 0 & 0 & 0 & 0 & 0 & 0 & 0 & 0 & 2.58 \cdot 10^{8} & 2.06 \cdot 10^{8} \\ 0 & 0 & 0 & 0 & 0 & 0 & 0 & 0 & 0 & 0 & 2.06 \cdot 10^{8} & 1.68 \cdot 10^{8}\end{array}\right]$

$\mathbf{D}_{\mathbf{0}}=\left[\begin{array}{cccccc}104.0 & 0 & 0 & 0 & -0.85 & 0 \\ 0 & 104.0 & 0 & 0.85 & 0 & 0 \\ 0 & 0 & 105.0 & 0 & 0 & 0 \\ 0 & 0.85 & 0 & 2.1 & 0 & 0 \\ -0.85 & 0 & 0 & 0 & 2.1 & 0 \\ 0 & 0 & 0 & 0 & 0 & 8.32\end{array}\right] \quad \mathbf{K}_{\mathbf{0}}=\left[\begin{array}{cccccc}117800 & 0 & 0 & 0 & 0 \\ 0 & 117800 & 0 & 0 & 0 \\ 0 & 0 & 169794 & 0 & 0 & 0 \\ 0 & 0 & 0 & 3279 & 0 & 0 \\ 0 & 0 & 0 & 0 & 3279 & 0 \\ 0 & 0 & 0 & 0 & 0 & 8685\end{array}\right]$

$\mathbf{B}=\left[\begin{array}{cccccc}0.577 & -0.577 & -0.789 & 0.789 & 0.211 & -0.211 \\ 0.577 & -0.577 & 0.211 & -0.211 & -0.789 & 0.789 \\ -0.577 & -0.577 & -0.577 & -0.577 & -0.577 & -0.577 \\ 0.086 & 0.077 & -0.110 & -0.113 & 0.023 & 0.036 \\ 0.077 & 0.086 & 0.036 & 0.023 & -0.113 & -0.110 \\ 0.163 & -0.163 & 0.163 & -0.163 & 0.163 & -0.163\end{array}\right]$

$\mathbf{R}_{\mathbf{1}}=\left[\begin{array}{cccccc}0.305 & -0.272 & -0.388 & 0.400 & 0.083 & -0.129 \\ 0.272 & -0.305 & 0.129 & -0.083 & -0.400 & 0.388 \\ -0.289 & -0.289 & -0.289 & -0.289 & -0.289 & -0.289 \\ 2.041 & 2.041 & -2.788 & -2.788 & 0.747 & 0.747 \\ 2.041 & 2.041 & 0.747 & 0.747 & -2.788 & -2.788 \\ 1.021 & -1.021 & 1.021 & -1.021 & 1.021 & -1.021\end{array}\right]$ 


\section{References}

[1] M. Heertjes, I. Sahin, N. van de Wouw, W. Heemels, Switching control in vibration isolation systems, IEEE Transactions on Control Systems Technology 21 (3) (2013) 626-635.

[2] K. Iwaya, R. Shimizu, T. Hashizume, T. Hitosugi, Systematic analyses of vibration noise of a vibration isolation system for high-resolution scanning tunneling microscopes, Review of Scientific Instruments 82 (8) (2011) 083702.

[3] S. Ito, D. Neyer, S. Pirker, J. Steininger, G. Schitter, Atomic force microscopy using voice coil actuators for vibration isolation, in: 2015 IEEE International Conference on Advanced Intelligent Mechatronics (AIM), IEEE, 2015, pp. 470-475.

[4] D. Karnopp, A. Trikha, Comparative study of optimization techniques for shock and vibration isolation, Transactions of American Society of Mechanical Engineers, Journal of Engineering for Industry 91 (4) (1969) 1128-1132.

[5] D. Tjepkema, J. van Dijk, H. Soemers, Sensor fusion for active vibration isolation in precision equipment, Journal of Sound and Vibration 331 (4) (2011) 735-749.

[6] A. Preumont, A. Franois, F. Bossens, A. Abu-Hanieh, Force feedback vs. acceleration feedback in active vibration isolation, Journal of Sound and Vibration 257 (4) (2002) 605-613.

[7] Y. Teo, A. Fleming, Optimal integral force feedback for active vibration control, Journal of Sound and Vibration 356 (2015) 20-33.

[8] A. Berkhoff, J. Wesselink, Combined mimo adaptive and decentralized controllers for broadband active noise and vibration control, Mechanical Systems and Signal Processing 25 (5) (2011) 1702-1714.

[9] S. Elliott, Optimal controllers and adaptive controllers for multichannel feedforward control of stochastic disturbances, IEEE Transactions on Signal Processing 48 (4) (2000) 1053-1060.

[10] D. Abramovitch, G. Hsu, Mitigating rotational disturbances on a disk drive with mismatched linear accelerometers, in: Proceedings of the Multi-Conference on Systems and Control, Sydney, NSW, Australia, 2015, pp. 1473-1478.

[11] L. Van de Ridder, W. Hakvoort, J. Van Dijk, Active vibration isolation control: comparison of feedback and feedforward control strategies applied to coriolis mass-flow meters, in: Proceedings of the American Control Conference, Chicago, IL, USA, 2015, pp. 2173-2178.

[12] E. Bjarnason, Analysis of the filtered-x lms algorithm, IEEE Transactions on Speech and Audio Processing 3 (6) (1995) 504514.

[13] S. Kuo, D. Morgan, Active noise control systems: algorithms and DSP implementations, John Wiley \& Sons, Inc., 1995.

[14] B. Widrow, S. Stearns, Adaptive signal processing, Englewood Cliffs, NJ, Prentice-Hall, 1985.

[15] J. Shynk, Adaptive iir filtering, IEEE ASSP Magazine 6 (2) (1989) 4-21.

[16] R. Fraanje, M. Verhaegen, N. Doelman, Convergence analysis of the filtered-u lms algorithm for active noise control in case perfect cancellation is not possible, Signal Processing 83 (6) (2003) 1239-1254.

[17] I. Landau, M. Alma, T. Airimitoaie, Adaptive feedforward compensation algorithms for active vibration control with mechanical coupling, Automatica 47 (10) (2011) 2185 - 2196.

[18] G. Williamson, S. Zimmermann, Globally convergent adaptive iir filters based on fixed pole locations, IEEE Transactions on Signal Processing 44 (6) (1996) 1418-1427.

[19] B. Ninness, J. Gómez, Frequency domain analysis of tracking and noise performance of adaptive algorithms, IEEE Transactions on Signal Processing 46 (5) (1998) 1314-1332.

[20] R. De Callafon, J. Zeng, C. Kinney, Active noise control in a forced-air cooling system, Control Engineering Practice 18 (9) (2010) 1045-1052.

[21] A. Sayed, Fundamentals of adaptive filtering, John Wiley \& Sons, 2003.

[22] E. Wan, Adjoint lms: An efficient alternative to the filtered-x lms and multiple error lms algorithms 3 (1996) 1842-1845.
[23] V. DeBrunner, D. Zhou, Hybrid filtered error lms algorithm: another alternative to filtered-x lms, IEEE Transactions on Circuits and Systems I: Regular Papers 53 (3) (2006) 653-661.

[24] S. Miyagi, H. Sakai, Mean-square performance of the filteredreference/filtered-error lms algorithm, IEEE Transactions on Circuits and Systems I: Regular Papers 52 (11) (2005) 24542463.

[25] L. Sujbert, A new filtered lms algorithm for active noise control, in: Proceedings of the International EAA Symposium on Active Control of Sound and Vibration, ACTIVE'99, 1999, pp. 11011110 .

[26] M. Beijen, J. van Dijk, W. Hakvoort, M. Heertjes, Self-tuning feedforward control for active vibration isolation of precision machines, in: Proceedings of The 19th IFAC World Congress, Cape Town, South Africa, 2014, pp. 5611-5616.

[27] D. Tjepkema, Active hard mount vibration isolation for precision equipment, Ph.D. thesis, University of Twente, Enschede, The Netherlands (2012).

[28] J. Jonker, R. Aarts, J. van Dijk, A linearized input-output representation of flexible multibody systems for control synthesis, Multibody Syst. Dyn. 21 (2009) 99-122.

[29] S. Kuo, J. Tsai, Residual noise shaping technique for active noise control systems, The Journal of the Acoustical Society of America 95 (1994) 1665-1668.

[30] H. Bao, I. M. S. Panahi, A novel feedforward active noise control structure with spectrum-tuning for residual noise, IEEE Transactions on Consumer Electronics 56 (4) (2010) 2093-2097.

[31] B. Widrow, E. Walach, Adaptive Inverse Control, Reissue Edition: A Signal Processing Approach, John Wiley \& Sons, Inc., 2008.

[32] S. Ishimitsu, S. Elliott, Improvement of the convergence property of adaptive feedforward controllers and their application to the active control of ship interior noise, Acoustical Science and Technology 25 (3) (2004) 181-187.

[33] A. Wang, W. Ren, Convergence analysis of the multi-variable filtered-x lms algorithm with application to active noise control, IEEE transactions on signal processing 47 (4) (1999) 1166-1169.

[34] R. Pintelon, J. Schoukens, System identification: a frequency domain approach, John Wiley \& Sons, 2012.

[35] R. Fraanje, S. Elliott, M. Verhaegen, Robustness of the filtered$\mathrm{x}$ lms algorithmpart ii: Robustness enhancement by minimal regularization for norm bounded uncertainty, IEEE Transactions on Signal Processing 55 (8) (2007) 4038-4047.

[36] G. Van der Poel, An exploration of active hard mount vibration isolation for precision equipment, Ph.D. thesis, University of Twente, Enschede, The Netherlands (2010).

[37] M. Seron, J. Braslavsky, G. Goodwin, Limitations in Filtering and Control, London: Springer-Verlag, 1997.

[38] M. Beijen, M. Heertjes, H. Butler, M. Steinbuch, Performance trade-offs in disturbance feedforward compensation of active hard-mounted vibration isolators, in: Proceedings of the American Control Conference, Chicago, IL, USA, 2015, pp. 21492154.

[39] M. Heertjes, B. Temizer, M. Schneiders, Self-tuning in masterslave synchronization of high-precision stage systems, Control Engineering Practice 21 (12) (2013) 1706-1715. 